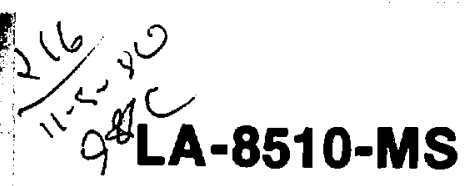

Informal Report

\section{Void Growth and Swelling for Cyclic Pulsed Radiation}

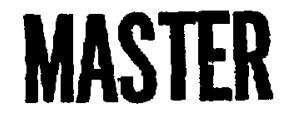

لِ

吾

章

章

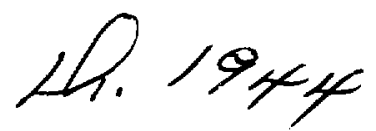

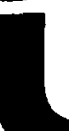


UC-25

Issued: September 1980

\section{Void Growth and Swelling for Cyclic Pulsed Radiation}

\author{
L. N. Kmetyk* \\ J. Weertman** \\ W. V. Green*** \\ W. F. Sommer
}

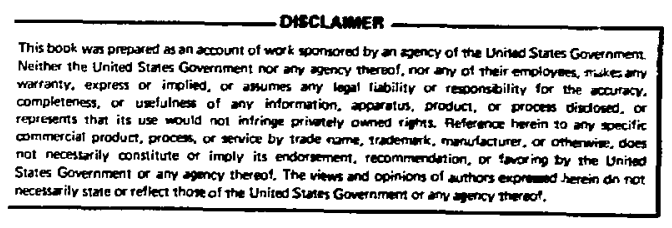

This book wa prepared as an acount of work somsored by an soency of the Unived Strites Government

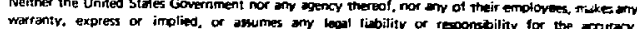

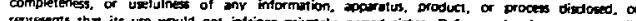
commer

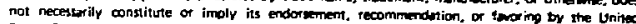

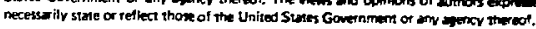


VOID GROWTH AND SWELLING FOR

CYCLIC PULSED RADIATION

by

L. N. Kmetyk, J. Weertman,

W. V. Green, and W. F. Sommer

\begin{abstract}
The analysis of Ghoniem and Kulcinski of a single radiation pulse has been extended to include the effects of temperature oscillations and multiple pulses by coupling six simultaneous nonlinear ordinary differential equations for point defect concentrations and sink strengths with a heat equation governing radiation-produced temperature fluctuations. The pulsed irradiation considered is that of the $800 \mathrm{MeV}$ proton beam at the clinton P. Anderson Meson Physics Facility (LAMPF), and numerical calculations have been done for aluminum and molybdenum. The model material is assumed to have been irradiated to a specific microstruciure, and the calculation is begun after the material has returned to thermal equilibrium conditions. Void growth is seen to proceed at a nearly linear rate after initlal transients (caused by the assumption of initial thermal equilibrium) in both vacancy concentration and vacancy loop size and number density. The temperature pulses associated with cyclic rather than steady irradiation generally enhance void growth if the ambient temperature is below the material peak swelling temperature, and conversely decreases void growth when the ambient temperature is above peak swelling temperature. The exception seen occurs when the temperature pulse is great enough that thermal emission of vacancies, rather than radiation production, is dominant.
\end{abstract}




\section{INTRODUCTION}

The fully dynamic rate theory, developed for steady state conditions by Brailsford and Bullough, was used by Kulcinski and Ghoniem to calculate void growth and swelling rates for a single radiation pulse impinging on a constant ambient temperature material. This theory is extended here to follow the effects of arbitrary numbers of repeated pulses and their associated radiationproduced temperature oscillations on material damage structure. The time dependence of such self-consistent temperature changes must be taken into account because the amount of void growth is a sensitive function of temperature. These temperature oscillations could also produce fluctuating thermoelastic and thermoplastic stresses which in turn would have an effect, and which have been neglected here.

Brailsford and Bullough [1] in 1972 first presented rate theory equations for spatially averaged point defect concentrations in irradiated materials containing voids, dislocation loops, deformation dislocations, and coherent or inculinle:t precipitates. Two coupled nonlinear algebraic equations, one for interstitial and the other for vacancy concentrations, were solved to yield a time-independent first approximation to the volume swelling rate for various ambient temperatures. After an incubation dose the volume swelling rate was found to have a linear dose dependence.

A rate theory for the growth of interstitial loops and the resulting irradiation creep in stressed irradiated material, containing voids, precipitates and network dislocations in addition to loops, was later presented [2]. The two coupled nonlinear algebraic equations for vacancy and interstitial consentrations were modified to include the effects of a tensile applied stress, which enhanced the vacancy emission rates and increased the nucleation rate for favorably oriented loops. These two equations were then solved to yield creep rates due both to the excess population of favored loops and to their faster growth rates.

The void growth rate theory was extended by Bullough, Eyre and Krishan [3] in 1975 to include the possibility of vacancy loop formation due to irradiationproduced cascade damage. Ordinary differential equatiuns (for void radius, interstitial loop radius, number density of vacancy loops, and fraction of vacancies tied up in vacancy loops) were added to the two algebraic point defect concentration equations, and were solved numerically for stress-free situations (although the equations derived were stress-dependent). The resulting 
theoretical swelling rates were used to force-fit the interstitial loop number observed by experiment, as a function of temperature.

The rate theory was extended to fully dynamic situations in 1976 by Ghoniem and Kulcinski [4,5] who replaced the two algebraic point defect concentration equations by analogous ordinary differential equations. The resultant six coupled nonlinear ordinary differential equations were solved numerically for steady irradiation and for a single pulse followed by a long annealing period. Although external uniaxial stress effects were included in the equations no results were given. Later reports $[6,7]$ in 1977 compared calculations of both steady-state irradiation void growth and thermal shrinkage of voids undergoing no irradiation to experimental data. Also investigated were the effect of collision cascade collapse and displacement rate on the growth aspect of voids in metal.

Dienes [8] studied special cases of the fully dynamic rate theory (with constant sink density and no recombination, and with zero sink density and constant recombination rate) which allowed analytic solutions of the two differential equations governing point defect concentrations. The concentration oscillations and their time integrals were compared for equivalent net doses of pulsed and steady irradiation.

In the present paper the six coupled simultaneous nonlinear ordinary differential equations solved by Kulcinski and Ghoniem are combined with a heat equation governing material temperature, whose source term includes both direct (radiation-produced lattice excitation) and indirect (annihilation energy of point defects) heating. This heat equation is shown to decouple from the ful1 set of equations only when indirect heating is negligible. Swelling rates in aluminum and molybdenum are calculated for samples irradiated in the LAMP $i$ proton beam, and the effect of varying pulse structure on swelling rates is investigated. (The LAMPF proton beam provides the only known source of pulsed irradiation where significant displacement damage energy levels can be diposited in metals.)

II. THEORY

We are given a material containing concentrations (expressed in number per lattice site, or atom fraction) $C_{i}$ and $c_{v}$ of free interstitials and vacincies, a concentration (expressed in number per unit volume) $N_{v}$ of voids of radius $r_{v}$, and a total dislocation drinsity $\rho_{d}$ given by 


$$
\rho_{\mathrm{d}}=\rho_{\mathrm{d}}^{0}+\rho_{\mathrm{d}}^{i \ell}+\rho_{\mathrm{d}}^{\mathrm{v} \ell}
$$

where $\rho_{\mathrm{d}}^{0}$ is the dislocation network line density, and $\rho_{\mathrm{d}}^{i l}$ and $\rho_{\mathrm{d}}^{\mathrm{v} \ell}$ the line densities of the irradiation produced interstitial and vacancy dislocation loops, given by

$$
\rho_{d}^{j, v \ell}=2 \pi r_{i, v}, N_{i, v \ell}
$$

$\mathrm{N}_{i}$ and $\mathrm{N}_{\mathrm{v}, \mathrm{n}}$ are the number per unit volume of interstitial and vacancy loops of radii $r_{i, j}$ and $r_{v i}$ :

These diclocation loops can change size in time by absorption of free vacancies and interstitials, and by thermal emission. The changes in the loop radii are given by

$$
\frac{d r}{d t} i^{n}=\frac{1}{b}\left\{Z_{i} D_{1} C_{1}-z_{v} D_{v} c_{v}+z_{v} D_{v} c_{v}^{0} \exp \left(\frac{-\left[_{s f}^{\gamma}+F_{e l}\left(r_{i l}\right)\right.}{k T} b^{2}\right)\right\}
$$

and

$$
\frac{d r}{d t} v^{0}=\frac{1}{b}\left\{z_{v} D_{v} C_{v}-z_{i} D_{i} C_{1}-z_{v} D_{v} c_{v}^{0} \exp \left(\frac{+\gamma_{s f}+F_{e 1}\left(r_{v \ell}\right)^{b} b^{2}}{k T}\right)\right\}
$$

where $D_{1}$ and $D_{v}$ are the diffusion coefficients respectively for interstitials and vacancies, $\gamma_{s f}$ is the stacking fault energy of the stacking fault of a dislocation (frop (set equal to zero if the loop contains no stacking fault), b is the length of the Burger's vector of a dislocation, $z_{v}$ is the dislocation bias factor for vacancies $\left(z_{v}=1\right)$ and $z_{i}$ is the bias factor for interstitials (slightly larger than one), $k$ is Boltzmann's constant and $T$ is the temperature, and $\mathrm{C}_{\mathbf{v}}{ }^{0}$ is the equilibrium vacancy concentration in an unradiated, unstressed metal. $F_{e l}$ is given by

$$
F_{e I}(r)=\frac{\mu b^{2}}{4 \pi(1-v)(r+b)} \log \left(\frac{r+b}{r}\right)
$$

where $r$ is the radius of an interstitial or vacancy loop $\left(r_{1 \ell}\right.$ or $\left.r_{v \ell}\right)$, $\mu$ is the shear modulus and $\nu$ is Poisson's ratio. Equation (5) represents the force on a unit length of dislocation that causes dislocation climb in the direction that 
decreases the loop radius $r$. The origin of this climb force is the self-energy or line tension of the dislocation loop.

In equations (3) and (4) a term analogous to the last term on the righthand-side of these equations has been dropped, since it contains the thermal equilibrium concentration $\mathrm{C}_{i}{ }^{0}$ of interstitials. Because the formation activation energy of interstitials generally is very high for all practical applications, the concentration $\mathrm{C}_{i}{ }^{0}$ is essentially identical to zero.

Vacancies produced by irradiation may be absorbed directly by voids, network dislocations or interstitial dislocations. Some of these vacancies, however, may first form vacancy dislocation loops, and as these loops shrink during climb, the vacancies are recreated. If $\varepsilon P(0<\varepsilon<1)$ represents the fraction of irradiation produced vacancies that initially condense into vacancy dislocation loops of radius $r_{v \ell}(0)$ per unit time, the number $n_{v \ell}$ of vacancy loops created in a unit volume per unit time is given by

$$
\mathrm{n}_{\mathrm{v} \ell}=\frac{\varepsilon \mathrm{Pb}^{2}}{\pi\left[\mathrm{r}_{\mathrm{v} \ell}(0)\right]^{2}}
$$

since there are $\frac{\pi b r_{v}^{2}}{b^{3}}$ vacancies in a dislocation loop of radius $r_{\ell}$. The average lifetime of a vacancy loop can be approximated by

$$
\tau \cong \frac{r_{v \ell}(0)}{\left.\frac{d r_{v \ell}}{d t}\right)_{0}}
$$

where $\left.\frac{d r v \ell}{d t}\right)_{0}$ is the value of equation (4) when $r_{v \ell}=r_{v \ell}(0)$, the initial radius of a vacancy dislocation loop.

If all the vacancies tied up in vacancy dislocation loops were released into the lattice, the vacancy concentration would be increased by an amount qu given by

$$
q_{v \ell}=\pi b r_{v \ell}{ }^{2} \mathrm{~N}_{v \ell}
$$

which can be rearranged to give 


$$
r_{v \ell}=\sqrt{\frac{q_{v \ell}}{\pi b N}}
$$

The rate of change of the number of vacancy loops per unit volume due to sascade collapse formation and shrinkage is given by

$$
\frac{d N_{v \ell}}{d t}=\frac{n_{v \ell}}{b^{3}}-\frac{N_{v \ell}}{\tau}
$$

which when combined with equations (4), (6), (7) and (8), becomes

$$
\begin{aligned}
\frac{d N_{v} \ell}{d t}=\frac{E P}{\pi b\left[r_{v \ell}(0)\right]^{2}} & -\frac{b}{r_{v}(0)}\left[Z v_{v} C_{v}-Z_{i} D_{i} C_{i}\right. \\
& \left.-Z_{v} D_{v} C_{v}^{0} \exp \left(\frac{+\left\{\gamma_{s f}+F_{e 1}\left[r_{v \ell}(0)\right]\right\} b^{2}}{k T}\right)\right]
\end{aligned}
$$

The time dependence of the fraction of vacancies tied up in vacancy loops, $q_{v \ell}$, is similarly given by

$$
\begin{aligned}
\frac{d q_{v \ell}}{d t}=\varepsilon P & -\sqrt{\frac{4 \pi q_{v \ell} v \ell}{b}}\left(z_{v} D_{v} C_{v}-z_{i} D_{i} C_{i}\right. \\
& \left.-Z_{v} D_{v} C_{v}^{0} \exp \left(\frac{+\left[\gamma_{s f}+F_{e 1}\left(r_{v \ell}\right)\right] b}{k T}\right\}\right)
\end{aligned}
$$

Free point defects are created at a rate $i$, annihilated by reconbination or absorbed by voids, edge dislocations or dislocation loops. The rate of change of the interstitial concentration $C_{i}$ is given by

$$
\frac{d c_{i}}{d t}=P-\alpha C_{i} C_{v}-C_{i} D_{i}\left\{4 \pi r_{v} N_{v}+z_{i}\left[\rho_{d}{ }^{0}+2 \pi\left(r_{i \ell} N_{i \ell}+r_{v \ell} N_{v l}\right)\right]\right\}
$$

where the recombination coefficient $a$ is given by 


$$
\begin{aligned}
\alpha & =g\left[v_{i} \exp \left(-\frac{E_{m}^{i}}{k T}\right)+v_{v} \exp \left(-\frac{E_{m}^{v}}{k T}\right)\right] \\
& =\left(g / b^{2}\right)\left(D_{i}+D_{v}\right) \approx g D_{i} / b^{2}
\end{aligned}
$$

where $E_{m}^{i}$ and $E_{m}^{v}$ are activation energies for migration of interstitials and vacancies respectively, $v_{i}$ and $v_{v}$ are frequencies associated with their motion, and $g$ is a constant of order of magnitude 1 . The term in equation (12) containing the constant $\alpha$ represents the destruction of interstitials by mutual annihilation with individual vacancies, while the terms proportional to the product $C_{i} D_{i}$ represent the elimination of interstitials at void sinks, dislocation network sinks and dislocation loop sinks.

The change in the vacancy concentration $C_{v}$ is similarly given by

$$
\begin{aligned}
& \frac{d C}{d t}=(1-\varepsilon) P-\alpha C_{i} C_{v}-C_{v} D_{v}\left\{4 \pi r_{v} N_{v}+Z z_{v}\left[\rho_{d}{ }^{0}+2 \pi\left(r_{i \ell} N_{i \ell}+r_{v \ell} N_{v \ell}\right)\right]\right\} \\
& +C_{v}{ }^{O} D_{v}\left\{4 \pi N_{v} r_{v} \exp \left[\left(\frac{2 Y}{r_{v}}-p_{g}\right) \frac{b^{3}}{k T}\right]+z_{v}\left[\rho_{d}^{0}+\right.\right. \\
& \left.2 \pi\left(r_{i \ell} N_{i \ell} \exp \left\{\frac{-\left[Y_{s f}+F_{e l}\left(r_{i \ell}\right)\right]_{b}^{2}}{k T}\right\}+r_{v \ell} N_{v \ell} \exp \left\{\frac{\left[Y_{s i}+F_{e l}\left(r_{v \ell}\right)\right]_{b}}{k T}\right\}\right)\right\}
\end{aligned}
$$

where $Y$ is the energy per unit surface of a void and $P_{g}$ is the pressure of the gas that may be present within a void. The terns in equation (14) that contain the constant $\alpha$ or the product $C_{v} D_{v}$ are analogous to similar terms in equation (12), while the terms that contain the product $C_{v}{ }^{0} D_{v}$ represent the continuous creation of vacancies by thermal emission at the voids, network dislacations and dislocation loops. (Note that any sink for point defects can also act as a source for point defects. Equation (12) should contain terms proportional to the product $C_{i}{ }^{0} D_{i}$, but because the value of $c_{i}^{0}$ is very small these terms can be dropped.) The gas pressure $\mathrm{p}_{\mathrm{g}}$ is given by

$$
p_{g}=\frac{n_{g} k T}{\frac{4}{3} \pi r_{v}^{3}-b n_{g}}
$$


where $n_{g}$ is the number of gas atoms or molecules within one void and $b_{v}$ is van der Waal's coefficient.

The rate of change of the void radius $r_{v}$ is given by

$$
\frac{d r_{v}}{d t}=\frac{I}{r_{v}}\left\{D_{v} C_{v}-D_{i} C_{i}-D_{v} C_{v}^{0} \exp \left[\left(\frac{2 Y}{r_{v}}-p_{g}\right) \frac{b^{3}}{k T}\right]\right\}
$$

where the first two terms represent absorption of tres potnt defects, and the last thermal emission of vacancies at the void surface.

If the swelling $S$ is defined as the change in volume of material divided by the initial volume of material, then the swelling is given by

$$
\mathrm{S}=\frac{4}{3} \pi \mathrm{r}_{\mathrm{v}}{ }^{3} \mathrm{~N}
$$

The inclusion of a fluctuating temperature $T(t)$ is quite simple, requiring only the substitution of this time-dependent temperature into equations (3), (10), (11), (13), (14), (15) and (16) where $T$ appears explicitly, as well as into the implicit temperature-dependent diffusion coefficients

$$
\mathrm{D}_{i, v}=\mathrm{D}_{i, v}^{0} \exp \left(-\frac{\mathrm{E}_{\mathrm{m}}^{\mathrm{i}, \mathrm{v}}}{\mathrm{kT}}\right)
$$

and the equilibrium vacancy concentration

$$
c_{v}^{o}=\exp \left(-\frac{E_{f}^{v}}{k T}\right)
$$

The temperature fluctuations can be determined from the particular irradiation loading condition specified. Suppose that a cylindrical target of radius $a$ and height $\ell$ is bombarded by $I(r, t)$ high energy particles per unit area per unit time, and assume that the particles are energetic enough and $\ell$ small enough that the irradiation damage is essentially uniform within the target in the direction of the beam. The temperature is found from the twodimensional time-dependent heat conduction equation 


$$
\frac{1}{K} \frac{\partial T}{\partial t}-\frac{1}{r} \frac{\partial}{\partial r}\left(r \frac{\partial T}{\partial r}\right)-\frac{\partial^{2} T}{\partial z^{2}}=\frac{1}{K} Q(r, t)
$$

where is is the thermal diffusivity and $K$ the thermel conductivity $(K=K \rho c$ where $\rho$ is the density of the material and $c$ its अecific heat).

The heat $Q(r, t)$ deposited per unit volume per unit itime by the flux $I(r, t)$ within the target can be divided into

$$
Q(r, t)=Q_{d}(r, t)+Q_{i}(r, t)
$$

The first part $Q_{d}(r, t)$ is the direct heat produced immediately within the bombarded region by excitation of the lattice. Thus, For the simplest case, $Q_{d}(r, t)$ is proportional to $I(r, t)$ and can be expressed as

$$
Q_{d}(r, t)=c_{d} I(r, t)
$$

where $c_{i}$ is the proportionality constant. The second part $Q_{i}(r, t)$ of the heat produced within the bombarded region is not realized immediately, but is the heat released. when point defects are destroyed, and is given by

$$
Q_{i}(r, t)=c_{d i}\left(P-\frac{\partial C_{i}}{\partial t}\right)+c_{d v}\left[(1-\varepsilon) P-\frac{\partial C v}{\partial t}\right]
$$

where $c_{d i}$ is the heat released by the destruction of a unit concentration of interstitials and $c_{d v}$ the same term for vacancies. The Frenkel pair production rate, $P$, is of course a Function of $I(r, t)$, and in the simplest case is proportional to $I$, giving

$$
P(r, t)=c_{p} I(r, t)
$$

where $c_{p}$ is the proportionality constant. Combining equations (12), (14), (21), (22) and (23) gives finally for the heat flow

$$
Q(r, t)=c_{d} I+c_{d i}\left\{\alpha C_{i} C_{v}+D_{i} C_{i}\left[4 \pi v_{v}^{N}+z_{i}\left(\rho_{d}{ }^{O}+2 \pi r_{i \ell} N_{i \ell}+2 \pi r_{v \ell} N_{v \ell}\right)\right]\right\}
$$




$$
\begin{aligned}
& +c_{d v}\left\{\alpha C_{v} C_{i}+D_{v} C_{v}\left[4 \pi r_{v} N_{v}+z_{v}\left(\rho_{d}{ }^{0}+2 \pi r_{i \ell}+2 \pi r_{v \ell}{ }^{N} v\right)\right]\right. \\
& +\mathrm{C}_{\mathrm{v}}{ }^{0} \mathrm{D}_{\mathrm{v}}\left[4 \pi \mathrm { r } _ { \mathrm { v } } \mathrm { N } _ { \mathrm { v } } \operatorname { e x p } \left(\left\{\frac{2 \gamma}{\mathrm{r}_{\mathrm{v}}}-i \mathrm{~g} / \frac{\mathrm{b}^{3}}{\mathrm{kT}}\right)\right.\right. \\
& +z_{v}\left(\rho_{d}{ }^{0}+2 \pi r_{i l} N_{i l} \exp \left[-\left\{\gamma_{s f}+F_{e 1}\left(r_{i l}\right)\right\} \frac{b^{2}}{k T}\right]\right. \\
& \left.\left.\left.+2 \pi r_{v \ell} v_{v \ell} \exp \left[\left\{r_{s f}+F_{e \ell}\left(r_{v \ell}\right)\right\} \frac{b^{2}}{i k T}\right]\right)\right]\right\}
\end{aligned}
$$

APPLICATION TO LAMPF PUISED BEAM

Consider a specific localized flux $I(r, t)$. The LAMPF $800 \mathrm{MeV}$ pulsed proton beam has an elliptically-shaped target area approximated here by acircular area. Since the flux profile is Gaussian, $I(r, t)$ is given by

$$
I(r, t)=I(t) \exp \left(-\frac{r^{2}}{r_{0}^{2}}\right)
$$

where $r_{0}$ is a constant giving the average sive of the bean: spot at the LAMPF facility and $I(t)$ is a pulsed time function, shown in figure 1 , equal to

$$
I(t)=\left\{\begin{array}{l}
0 \quad \text { for } t<0 \\
I_{0} \text { for } n t^{*} \leq t \leq n t^{*}+\Delta t \\
0 \text { for } n t^{*}+\Delta t \leq t \leq(n+1) t^{*} \\
\text { where } m \leq t \leq m \tau+\tau_{1} \\
0 \quad \text { for } m \tau+\tau_{1} \leq t \leq(m+1) \tau
\end{array}\right.
$$

where $n, m=0,1,2, \ldots$ and $I_{\mathrm{f}}$ is a constant. If $n_{p}$ is the total number of $800 \mathrm{MeV}$ protons in a pulse of duration $\Delta t$, then $I_{0}$ is given by

$$
I_{0}=\frac{n_{p / \Delta t}}{\int_{0}^{a} 2 \pi r \exp \left(\frac{-r^{2}}{r_{0}^{2}}\right) d r} \cong \frac{n p / \Delta t}{\pi r_{0}^{2}}
$$


The LAMPF accelerator operates at a frequency $\left(\tau^{-1}\right)$ of $120 \mathrm{~Hz}$ with pulse bundles $0.5 \mathrm{~ms}$ long $\left(\tau_{l}\right)$, containing $0.25 \mathrm{~ns}$ ( $\Delta \mathrm{t}$ ) pulses occurring once every $5 \mathrm{~ns}\left(\mathrm{t}^{*}\right)$. Thus the average proton flux (i.e., the macropulses) is on 6 percent of the time. (For comparison, calculations will be made with other percent "on-times" ranging from 0.1 percent to 100 percent. The frequency and total damage per pulse will be held constant; the pulse length and anuealing time will be adjusted accordingly.)

Suppose each $800 \mathrm{MeV}$ proton loses an amount of energy $E^{*}$ per unit distance traversed in the target, so that if one $800 \mathrm{MeV}$ proton hits a unit area of the target it gives up the same energy $E^{*}$ per unit volume of material. of this 1ost energy a fraction $\alpha^{*}$ goes directly into heat through lattice excitation and the other fraction $\left(i-\alpha^{*}\right)$ goes into point defect production. Thus $c_{d:}$ is given by

$$
c_{d}=c^{*} E^{*}
$$

The proportionality constant $c_{p}$ that appears in the Frenkel pair production rate given by equation (24) is found by noting that the energy lost by an 800 MeV proton which does not immediately appear as heat is stored in the point defect formation energy, so that

$$
c_{p}=\frac{\left(1-\alpha^{*}\right) E^{*} b^{3}}{E_{f}^{i}+E_{f}^{v}}
$$

where $E_{F}^{i}$ and $E_{f}{ }^{v}$ are again the formation energies respectively of interstitiais and vacancies. (The term $b^{3}$ insures that the generation rate $P(r, t)$ is expressed in units of atom fraciion per second.)

The fraction $\alpha^{*}$ of energy appearing directly as heat is found by equating the above expression with the standard point defect production rate [9]

$$
P(r, t)=\frac{.8 \sigma_{D}}{2 E_{D}} I(r, t)=c_{p} I(r, t)
$$

in which $\sigma_{D}$ is tine damage energy cross section in b-keV and $E_{D}$ the threshold energy in $\mathrm{eV}$. Once $\alpha^{*}$ is found, the proportionality constant $c_{\mathrm{d}}$ is also 
determined, and the total heat generation per unit time per unit volume in each indiridual pulse is given by

$$
Q(r, t)=c_{d} I(r, t)=\alpha^{*} E^{*} I_{0} \exp \left(-\frac{r^{2}}{r_{0}^{2}}\right)
$$

The heat released by the destruction of a unit concentration of interstitials ( $c_{d i}$ ) or vacancies $\left(c_{d v}\right)$ used in equation (23) is given by

$$
c_{d i, v}=E_{f}^{i, v} / b^{3}
$$

\section{APPROXIMATIONS}

Several special cases of the fully dynamic rate thecry can be studied analytically for temperature fluctuations so small they can be neglected. One such case is that of constant recombination rate and zero sink density. The other, studied here, is that of zero recombination and constant total sink density.

The point defect concentration equations (12) and (14) become, if $\alpha$ is set equal to zero,

$$
\begin{aligned}
& \frac{d C_{i}}{d t}=P-D_{i} C_{i} \rho_{\text {sink }} \\
& \frac{d C_{v}}{d t}=(1-\varepsilon) P-D_{v} C_{v} \rho_{\text {sink }}+P_{t h}
\end{aligned}
$$

where $\rho_{\text {sink }}$ is the total sink density given by

$$
\rho_{\text {sink }}=4 \pi r_{v} N_{v}+\rho_{d}^{0}+2 \pi\left(r_{i \ell} N_{i \ell}+r_{v \ell} N_{v \ell}\right)
$$

and $P_{t h}$ is the rate of thermal emission of vacancies from the various sinks, given by 


$$
\begin{aligned}
p_{t h}= & c_{v}^{0} D_{v}\left\{4 \pi N_{v} r_{v} \exp \left[\left(\frac{2 \gamma}{r_{v}}-p_{g}\right) \frac{b^{3}}{k T}\right]+z_{v}\left[\rho_{d}^{0}\right.\right. \\
& +2 \pi\left(N_{i \ell} r_{i \ell} \exp \left\{\frac{-\left[\gamma_{s f}+F_{e 1}\left(r_{i{ }^{\prime}}\right)\right] b^{2}}{k T}\right\}\right. \\
& \left.\left.+N_{v \ell} r_{v \ell} \exp \left\{\frac{+\left[\dot{\gamma}_{s f}+F_{e 1}\left(r_{v \ell}\right)\right] b^{2}}{k T} \mid\right)\right]\right\}
\end{aligned}
$$

With all the sink properties held constant the solution of these approximate point defect concentration equations for the time during wich the material is being irradiated is

$$
\begin{aligned}
& \begin{aligned}
C_{i}\left(k \tau \leq t \leq k \tau+\tau_{I}\right)= & \left.\frac{P}{J_{i} \rho_{\text {sink }}}\left\{1-\exp \mid-D_{i} \rho_{\text {sink }}(t-k \tau)\right]\right\} \\
& +C_{i}(k \tau) \exp \left[-D_{i} \rho_{\text {sink }}(t-k \tau)\right] \\
C_{v}\left(k \tau \leq t \leq k \tau+\tau_{1}\right)= & \frac{(1-\varepsilon) P+P_{t h}}{D_{v} \rho_{\text {sink }}}\left\{1-\exp \left[-D_{v} \rho_{\text {sink }}(t-k \tau)\right]\right\} \\
& +C_{v}(k \tau) \exp \left[-D_{v} \rho_{\text {sink }}(t-k \tau)\right]
\end{aligned}
\end{aligned}
$$

The initial conditions for $k=0$ are taken to be $c_{i}(t=0) \equiv 0$ (since $c_{i}^{0}$ is very sma11) and $c_{v}(t=0)=c_{v}^{0}$, the termal equilibrium concentration of vacancies. The solution for the annealing period is similarly

$$
\begin{aligned}
C_{i}\left[k \tau+\tau_{1} \leq t \leq(k+1) \tau\right]= & C_{i}\left(k \tau+\tau_{1}\right) \exp \left[-D_{i} \rho_{\operatorname{sink}}\left(t-k \tau-\tau_{1}\right)\right] \\
C_{v}\left[k \tau+\tau_{1} \leq t \leq(k+1) \tau\right]= & \frac{P_{t h}}{D_{v} \rho_{\operatorname{sink}}}\left\{1-\exp \left[-D_{v} \rho_{\operatorname{sink}}\left(t-k \tau-\tau_{1}\right)\right]\right\} \\
& +C_{v}\left(k \tau+\tau_{1}\right) \exp \left[-D_{v} \rho_{\text {sink }}\left(t-k \tau-\tau_{1}\right)\right]
\end{aligned}
$$


For many situations the recombination rate is negligible compared to the sink absorption rate, and the change in sink densities and sizes (and thus total strength) is small for any given pulse. The approximate expressions derived above are then valid, and can be used to study the small changes in various sink properties during and between single pulses, although repeated applications to multiple pulses lead tc large cumulative errors. The void radius equation (16) con be written as

$$
\frac{d r}{d t}=\frac{1}{r_{v}}\left(D_{v} C_{v}-D_{i} C_{i}-P_{t h}^{v}\right)
$$

where $P_{t h}^{v}$ represents the thermal emission of vacancies at the void surface given by

$$
\mathrm{p}_{\mathrm{th}}^{\mathrm{v}}=\mathrm{D}_{\mathrm{v}} \mathrm{C}_{\mathrm{v}}^{0} \exp \left[\left(\frac{2 \gamma}{\mathrm{r}_{\mathrm{v}}}-\mathrm{p}_{\mathrm{g}}\right) \frac{\mathrm{b}^{3}}{\mathrm{kT}}\right]
$$

If the thermal emission term is assumed constant (neglecting the effect of changing $r_{v}$ and indirectly $p_{g}$, which is given in equation (15) as a function of $r_{v}$ ), the solution of equation (42) for the irradiation periods, found by substituting equations (38) and (39) for $c_{i}$ and $c_{v}$ and integrating, is

$$
\begin{aligned}
& r_{v}^{2}\left(k t \leq t \leq k \tau+\tau_{1}\right) \quad{ }_{v}^{2}(k \tau)+2\left(\frac{P+p_{t h}}{\rho_{\text {sink }}}-p_{t h}^{v}\right)(t-k \tau) \\
& \quad+\frac{2}{\rho_{\text {sink }}}\left[C_{i}\left(k \tau \leq \tau \leq k \tau+\tau_{1}\right)-C_{i}(k \tau)-C_{v}\left(k \tau \leq t \leq k \tau+\tau_{1}\right)+C_{v}(k \tau)\right]
\end{aligned}
$$

The change in void radius during the annealing period is likewise found by substituting equations (40) and (41) for $C_{i}$ and $C_{v}$ into equation (42) and integrating, and is given by 


$$
\begin{aligned}
r_{v}^{2}[k \tau & \left.+\tau_{1} \leq t \leq(k+1) \tau\right]=r_{v}^{2}\left(k \tau+\tau_{1}\right)+2\left(\frac{P_{t h}}{\rho_{\text {sink }}}-p_{t h}^{v}\right)\left(t-k \tau-\tau_{1}\right) \\
& +\frac{2}{\rho_{\text {sink }}}\left\{c_{i}\left[k \tau+\tau_{1} \leq \tau \leq(k+1) \tau\right]-c_{i}\left(k \tau+\tau_{1}\right)\right\} \\
& -\frac{2}{\rho_{\text {sink }}}\left\{c_{v}\left[k \tau+\tau_{1} \leq t \leq(k+1) \tau\right]+c_{v}\left(k \tau+\tau_{1}\right)\right\}
\end{aligned}
$$

The interstitial loop radius, described by equation (3), is similarly approximated during the irradiation and annea! ing periods by the equations

$$
\begin{aligned}
& \left.r_{i \ell(k \tau} \leq t \leq k \tau+\tau_{1}\right)=r_{i \ell}(k \tau)-\frac{1}{b}\left(\frac{P+P_{t h}}{\rho_{\text {sink }}}-P_{t h}^{i \ell}\right)(t-k \tau) \\
& -\frac{1}{b \rho_{\text {sink }}}\left[z_{i} c_{i}\left(k \tau \leq t \leq k \tau+\tau_{1}^{\prime}\right)-z_{i} c_{i}(k \tau)\right]- \\
& -\frac{1}{b \rho_{\text {sink }}}\left[-z_{v} c_{v}\left(k \tau<t<k \tau+\tau_{1}\right)+z_{v} c_{v}(k \tau)\right]
\end{aligned}
$$

and

$$
\begin{aligned}
& \mathrm{r}_{i \ell l}\left[k \tau+\tau_{1} \leq t \leq(k+1) \tau\right]=\mathrm{r}_{\dot{i} \ell}\left(k \tau+\tau_{1}\right)-\frac{1}{b}\left(\frac{\rho_{t h}}{\rho_{\text {sink }}}-P_{t h}^{i \ell}\right)(t-k \tau-\tau 1) \\
& -\frac{1}{b \rho_{\operatorname{sink}}}\left\{z_{i} c_{i}\left[k \tau+\tau_{1} \leq t \leq(k+1) \tau\right]-z_{i} C_{i}\left(k \tau+\tau_{1}\right)\right\} \\
& -\frac{1}{b \rho_{\operatorname{sink}}}\left\{-z_{v} C_{v}\left[k \tau+\tau_{1} \leq t \leq(k+1) \tau\right]-z_{v} C_{v}\left(k \tau+\tau_{1}\right)\right\}
\end{aligned}
$$

where $p_{t h}^{i l}$ represents the enirssion of vacancies at the surface of the interstitial loops, given by

$$
p_{t h}^{i \ell}=D_{v} c_{v}^{0} \exp \left\{\frac{-\left[Y_{s f}+F_{e l}\left(r_{i \ell}\right)\right] b^{2}}{k T}\right\}
$$


The vacancy loop properities, described by equations (10) and (11), are slightly more complicated. The vacancy loop number density equation is solved as an example. During the irradiation period the vacancy loop number density is given by

$$
\begin{aligned}
& N_{v \ell}\left(k \tau \leq t \leq k \tau+\tau_{1}\right) \\
&=N_{v \ell}(k \tau)-\frac{b}{r_{v \ell}(0)}\left(\frac{p+p_{t h}}{\rho_{s i n k}}-\left\{p_{t h}^{v \ell}\left[r_{v \ell}(0)\right]+\frac{\varepsilon P}{\pi b^{2} r_{v \ell}(0)}\right\}\right)(t-k \tau) \\
&-\frac{b}{r_{v \ell}(0)}\left[z_{i} c_{i}\left(k \tau \leq t \leq k \tau+\tau_{1}\right)-z_{i} c_{i}(k \tau)\right] \\
&-\frac{b}{r_{v \ell}(0)}\left[-z_{v} c_{v}\left(k \tau \leq t \leq k \tau+\tau_{1}\right)+z_{v} c_{v}(k \tau)\right]
\end{aligned}
$$

where the thermal emission of vacancies at the surface of the vacancy loop is given by

$$
p_{t h}^{v \ell}=D_{v} c_{v}^{0} \exp \left\{\frac{+\left[\gamma_{s f}+F_{e l}\left(r_{v \ell}\right)\right] b^{2}}{k T}\right\}
$$

The vacancy loop number density during the annealing period is given by

$$
\begin{aligned}
& \mathrm{N}_{\mathrm{v} \ell}\left[\mathrm{k \tau}+\tau_{1} \leq \mathrm{t} \leq(\mathrm{k}+1) \tau\right] \\
& =N_{v \ell}\left(k \tau+\tau_{1}\right)-\frac{b}{r_{v \ell(0)}}\left(\frac{p_{t h}}{\rho_{\text {sink }}}-\left\{p_{t h}^{v \ell}\left[r_{v \ell}(0)\right]+\frac{\varepsilon P}{\pi b^{2} r_{v \ell(0)}}\right\}\right)\left(t-k \tau-\tau_{1}\right) \\
& -\frac{b}{r_{v \ell}(0)}\left\{z_{f} C_{i}\left[k \tau+\tau_{1} \leq t \leq(k+1) \tau\right]-z_{i} C_{i}\left(k \tau+\tau_{1}\right)\right\} \\
& -\frac{b}{r_{v \ell}(0)}\left\{-z_{v} c_{v}\left[k \tau+\tau_{1} \leq t \leq(k+1) \tau \mid+z_{v} C_{v}\left(k \tau+\tau_{1}\right)\right\}\right.
\end{aligned}
$$


The expressions giving $\mathcal{F}_{\mathrm{v} l}$ are much more elaborate and are not given here.

IV. NUMERICAL SOLUTION

Equations (3), (10), (11), (12), (14), (16) and (20) constitute a system of seven non-linear interdependent first order differential equations. The indirect heating term in the temperature equation, which would normally couple the six ordinary differential equations for $r_{i \ell}, \mathbb{N}_{v \ell}, q_{v \ell}, C_{i}, C_{v}$, and $r_{v}$ to a partial. differential equation for $T$, is shown in Appendix $I$ to be negligible in the situations considered in this paper. The temperature equation thus completely decouples from the other six equations, and can be solved analytically, as discussed in detail in Appendix II. Since this analytic solution provides the temperature at any spatial point as a function of time, the problem reduces back to a system of ordinary rather than partial differential equations. One of the difficulties of solving time-dependent materials problems such as this is the "stiffness" of the resulting system of equations, requiring special numerical techniques.

A system of ODE's is generally regarded as being stiff if it involves both very rapidly changing terms and very slowly changing terms, all of a decaying nature, so that it models a physical system with widely differing time constants. More precisely, consider the eigenvalues $\lambda_{i}$ of the NxN Jacobean matrix

$$
\left.J=\frac{\partial f_{i}}{\partial y_{j}}\right)_{i, j=1}
$$

and suppose that a11 the $\lambda_{i}$ have negative real parts. If the time constants

$$
1 /\left|\operatorname{Re}\left(\lambda_{i}\right)\right|, 1 /\left|\operatorname{Re}\left(\lambda_{2}\right)\right|, \ldots, 1 /\left|\operatorname{Re}\left(\lambda_{N}\right)\right|
$$

are widely varying, the system is called stiff. Since $J$ varies with time the eigenvalues do, too, and consequently stiffness is a local property. A system may be stiff in some regions of $t$ and not in others. Stiffness is also a relative property, measured by the ratio of the maximum to minimum time constants, $\left(\max \tau_{i} / \min \tau_{i}\right)$. The difficulty in solving stiff problems is that, while $\pi$.st conventional ODE solvers require time increments commensurate with $m i n \tau_{i}$, the 
size $\left|t-t_{0}\right|$ of the problem range is commensurate with max $\tau_{i}$. Thus, the problem cannot run to completion in a reasonable number of steps.

The numerical method used is based on ideas of C. W. Gear [10] and A. Hindmarsh [11] for the solution of the initial-value problem for systems of ODE's. The package of subroutines SDRIVE [12] includes both the implicit Adams method and the linear multistep methods, or backwards differentiation formulas, of Gear as options. With Gear's methods, the increment $h$ is restricted to small values (by the requirements of accuracy), only where the solution is relatively active. Since by definition the problem is not stiff in such regions, accuracy is achieved at minimum cost by allowing both $h$ and the order of the method to vary. In regions of stiffness where the solution is inactive, Gear's methods have the property of "stiff stability", which assures that $h$ is no longer restricted by the small time constants unless or until the corresponding rapidly decaying terms again become active. Among other things, this property requires that the method be implicit and a system of generally non-linear algebraic equations be solved at each step. Stiffness, moreover, means that a fairly powerful iteration method (variants of Newton's methods called chord methods) must be used to solve this system.

The main program, HANS, first sets all the required material constants and the parameter options for the equation solver SDRIVE (e.g., number of equations, method of solution, error control, etc.), as well as the initial conditions given, Then, for each pulse and each annealing pericd, the subroutine package SDRIVE is called. When return occurs, either the solution and the numerical statistics are printed out and the next integration is begun, or (if an error occurs during the attempted integration) an error message is printed and the program stops.

Subroutine DIFFUN, required by the SDRIVE package, calculates $\dot{y}=f(y, t)$ for the irradiation period. An analogous subroutine, DIFF, calculates $y=f(y, t)$ for the annealing periods.

The irradiation load itself is calculated in function BEAM. If the irradiation is constant or slowly varying in time, the integration routine's usual mode of solution, using the largest possible (and hence most effictent) choice of time-step to overshoot and then interpolating back to desired ovtput times, can be used with no difficulty. However, if a singularity exists beyond the desired solution time (as would occur, for example, when the irradiation load consists of a step function as described earlier), the solution must be 
obtained by integrating exactly to the desired time point, limiting the variable time-step to the order of magnitude of the characteristic pulse time and thus increasing the computer time required for solution.

\section{RESULTS}

The fully dynamic rate theory with self-consistent temperature effects described above has been used to analyze the behavior of both aluminim and molybdenum during cyclic pulsed radiation and to compare the results with those for equivalent dose steady state irradiation. The base case pulsed irradiation is taken to be that of the LAMPF $800 \mathrm{MeV}$ proton beam.

The beam at LAMPF is assumed to have a Gaussian radial flux profile given by equation (26). Its stepwise time function, described in equation (27), consists of $0.25 \mathrm{~ns}$ micropulses occurring every 5 ns in macropulse bundles $0.5 \mathrm{~ms}$ wide at a frequency of $120 \mathrm{~s}^{-1}$, as shown in Figure 1 . To see whether this structure must be taken into account, the various pulse times must be compared to the characteristic time constants fo the differential equations. (If the pulsing is much more rapid than the variables' reaction times, the system will not see any new effects.) The vacancy and interstitial characteristic reaction times (the shortest in the system) can be approxinated by

$$
\tau_{i, v}=D_{i, v} \rho_{\text {sink }}
$$

from equations (34) and (35). For the material data given in Tables 1 and 2 this leads to the ordering

$$
\begin{aligned}
& \Delta t, t * \ll \tau_{i} \tau_{v} \\
& \tau_{i} \ll \tau_{1} \quad \tau \quad \tau \tau \ll \tau_{v}
\end{aligned}
$$

The sample cannot react to the micropulses, but is affected by the macropulses. To test whether the beam microstructure can in fact be neglected, two calculations were done, whose results are shown in Fig. 2. In the first calculation, the first $10^{-4} \mathrm{~s}$ of a macropulse was run with its $2 \times 10^{4}$ micropulses modelled accurately, while in the second an average macropulse was used. The material was taken to be aluminum at an ambient temperature of $400 \mathrm{~K}$. The point defect 
concentracions arealmost identical, and confirm that the LAMP bean microstructure is indeed negligible. Therefore, all following calculations have only average macropulses modelled.

The concentrations shwon in Fig. 2 are for the center point of a thin cylindrical target, as described in Appendix $I$, The radiation damage due to high energy particles such as $800 \mathrm{MeV}$ protons is uniform in depth for thin samples. The effect of the Gaussian radial flux profile is shown in Fig. 3. The vacancy concentration is simply proportional to the current at any given location, since there has not been time for any diffusion to occur. The interstitals, being much more mobile, as seen in equation (53), have reached an equilibrium concentration early in the pulse $\left(\sim 10^{-6} \mathrm{sec}\right)$. The enhanced diffusion due to higher radiation produced temperatures at the sample center causes the equilibrium concentration there to decrease, leading to interstitial concentration profiles peaked at some intermediate radius. The vacancy concentration is peaked at the sample center, and both the vacancy and interstitial concentrations are within a few percent of the values predicted by equations (38) - (41). The interstitial concentration very rapidly decays back to thermal equilibrium at the end of the pulse, while the vacancy concentration remains constant during the annealing period, also as predictec by the approximate equations.

The vacancy concentration will not increase linearly with time (as in Fig. 3) indefinitely, but will also come to an equilibrium concentration after many pulses, as shown in Fig. 4. The vacancy concentration oscillates between 1 imits, whose separation depends on the relative duration of the irradiation and annealing periods. For the LAMPF beam macrostructure the fractional "on-time" or $\frac{\tau_{1}}{\tau}(=\mathrm{f}$ ) is 0.06 . (The pulse structure in this study is varied by keeping $\tau$ fixed to the LAMPF value of $1 / 120$ seconds, and changing $\tau_{1}$. While the vacancy concentration is approaching its equilibrium value, the voids are primarily absorbing interstitials and hence shrinking, as shown in Fig. 4. As the vacancy concentration stabilizes, the voids begin to grow at a nearly uniforn rate. This initial vold shrinkage is also well predicted by the approxinate sequence of pulses. Molybdenum requires many more pulses than aluminum before the vacancy concentration Iimits are reached, because of its lower vacancy diffusivity. The length of this initial void shrinkage is also a sensitive function of the ambient temperature, which strongly influences the diffusion rate - the higher the temperature, the fewer pulses required for the vacancy concentration to saturate. 
A second and much slower initial "transient" is shown in Figure 5. The vacancy loops take approximately ten times as many pulses to reach an eventual oscillating steady state for both size and number density as does the vacancy concentration. (The oscillations are too small to be seen on the scale of Figure 5.) The final value of the vacancy loop average radius ( $3 A$ ) represents a small cluster of vacancles rather than a true vacancy dislocation loop. This illustrates a defect in the theory, that no term is present to correctly account for the disappearance of vacancy loops as they shrink down to individual vacancies.

When the vacancy loop size and number density reach equilfbrium values, void growth rate becomes constant as shown in Figure 6. The vioid growth rat: will change only gradually under further irradiation, as shown in Figure 7 , as the total sink strength slowly increases. The approximate equations gave excellent agreement for short sequences of pulses when initial conditions are taken from the numerical calculations, but completely fail to duplicate the results in Figures 6 and 7 , due to cumulative error, if they are iterated for many pulses.

The above calculations were repeated with the tenperature pulsing suppressed. Since Dienes' work [8] concluded that it was the nonlinear recombination term that would be responsible for the difference between steady state and cyclic pulsed irradiation behavior, the calculations were also rerun with no recombination $(\alpha=0)$, with the results shown in Figure 8. Nearly identical void growth rates are obtained in the presence or absence of recombination, and a similar lack of sensitivity on very small temperature excursions is seen. The calculated results only show a significantly lower void growth rate when the "on time" is very siort, the temperature excursion large and the displacement damage rate very large for a short period of tine. (The maximum temperature rise for the various pulse structures considered is given in table 3 ).

The ambient temperature used in the above calculation $\left(T_{0}=400 \mathrm{~K}\right)$ is actually above the peak swelling temperature for the "simulated Al", as is shown in Fig. 9. We expect that temperature pulsing would produce enhanced void growth if the ambient temperature were below the peak swelling temperature. A series of calculations for aluminum at an ambient temperature of $352 \mathrm{~K}$ did indeed show this effect, as seen in in Fig. 10. The early void shrinkage is not shown, but both the vacancy concentration and the vacancy loop size and number 
density transients were present, and lasted much longer than their analogs in $400 \mathrm{~K}$ aluminum, due to the decrease in vacancy diffusivity at lower temperatures.

A parallel set of calculations was done for a molybdenum target. Ambient temperatures of $1200 \mathrm{~K}$ and $1600 \mathrm{~K}$ were chosen as bracketing the peak swelling temperature, as shown in Fig. 11. The lower temperature calculations $\left(\mathrm{T}_{0}=\right.$ 1200 i) gave the expected result of increasing temperature pulsing increasing void growth, as shown in Fig. 12, but the higher temperature calculations $\left(\mathrm{T}_{0} \times 1600 \mathrm{~K}\right)$ gave contradictory results, with the voids growing faster than more slowly for $f=0.1 \%$ than for $f=1 \%$, as seen in Fig. 13. Upon investigation, the temperature excursion for the short pulse length was found to be so large that, when added to the high amblent temperature, the thermal emission of vacancies dominated the radiation production during the latter part of each pulse. Thermal emission also remalned important during the early part of each annealing period. The result was, as seen in Fig. 14, to decrease the total sink density as a function of time rather than to increase it, and hence to increase the void growth rate.

\section{CONCLUSIONS}

The fully dynamic rate theory developed by Ghoniem and Kulcinski has been used to study the behavior of both aluminum and molybdenum under cyclic pulsed irradiation. Several important conclusions can be drawn:

(1) The pulsed nature of irradiation is important only when the characteristic pulse times are greater than or comparable to the vacancy and interstitial characteristic reaction times (the shortest in the system). If the pulsing is much more rapid than the varlables' reaction time, the system will not see any new effects.

(2) The assumption that the material initially contains a given microstructure and is at thermal equilibrium results in transients in both vacancy concentration and vacancy loop size and number density. Since vold growth does nit proceed at a falrly linear rate until these transients have ended, single or few pulse behavior cannot be extrapolated to estimate long-term behavior, as sas done by Ghoniem and Kulcinski.

(3) Pulsing the irradiat Ion has only an indirect effect on material swelling.

(a) When the cyclic radiation is assumed to produce self-consistent 
temperature pulses, the material "sees" enhanced ambient temperatures. This temperature enhancement increases void growth rate if the amblent temperature is below the material peak swelling temperature, and conversely decreases vold growth rate when the amblent temperature is above peak swelling temperature.

b) When radiation-produced temperature pulses are suppressed so that the material remains at a constant ambient temperature, the void growth rate does not change as the radiation is fragmented into varlous pulse structures. Although Dlenes' analytlcal work concluded that the nonlinear recombination term would be affected by the pulsed nature of the irradiation, our calculations did not confirm this. In the absence of recombination, the void growth rate was greater since vacancles formerly destroyed by recombination were now available for absorption by volds, but no effect was seen as the pulse structure was varied.

(4) For high ambient temperatures and short intense radiation pulses, which cause large temperature excursions, thermal emission of vacancies greatly overshadows radiation production during the latter part of each pulse, and the general effect of pulse structure and ambient temperature on void growth summarized above is no longer applicable.

\section{ACKNOWLEDGMENTS}

This work was performed under the auspices of the Department of Energy. The authors wish to express their appreciation to Dr. R. J. Bard for interest, encouragement and support and to Dr. D. M. Parkin for many helpr̂ul discussions. 
The heat $Q$ deposited per unit time per unit volume in the target by a flux I consists of the direct heat produced immediately within -the bombarded region by excltation of the lattice, and the heat released when point defects are destroyed by recombination or absorption at sinks. The second term is in most applications negligible.

The direct heat is given by

$$
Q_{d}=E^{*} I
$$

where $E^{*}$ is the amount of energy lost by an $800 \mathrm{MeV}$ proton per unit distance traversed in the target. (We have assumed $\alpha * \approx 1$. ) The 1ndirect heating term is

$$
Q_{i}=\frac{E_{f}^{i}}{b^{3}}\left(P-\frac{\partial C_{i}}{\partial t}\right)+\frac{E_{f}^{v}}{b^{3}}\left[(1-\varepsilon) P-\frac{\partial C_{v}}{\partial t}\right]
$$

where $\frac{E_{f}^{i, v}}{b^{3}}$ is the heat released by the destruction of a unit concentration of interstitials or vacancies with formation energies $E_{f}{ }^{i, v}$. Using equations (12) and (13), this expression can be rewritten as

$$
Q_{i}=\frac{E_{f}^{i}}{b^{3}}\left(\alpha C_{i} C_{v}+D_{i} C_{i} \rho \operatorname{sink}\right)+\frac{E_{f}^{v}}{b^{3}}\left(\alpha C_{i} C_{v}+D_{v} C_{v} \rho \operatorname{sink}-P_{t h}\right)
$$

where $\rho_{\text {sink }}$ is the total sink density defined in equation (36).

The maximum possible values that $C_{i}$ and $C_{v}$ can have are, fron equations (38) - (41),

$$
\begin{aligned}
& c_{i}=\frac{P}{D_{1} \rho_{\text {sink }}} \\
& c_{v}=\frac{(1-\varepsilon) P+P_{t h}}{D_{v} \rho_{\text {sink }}}
\end{aligned}
$$


If the total sink density is assumed constant and if the the rmal emission of vacancles is neglected (a good assumption for the conditions we are interested $i n)$, the direct and indirect heating for the aluminum and molybdenum samples described in Tables 1 and 2 are

\begin{tabular}{|c|c|c|c|c|}
\hline & $325 \mathrm{~K}$ & $400 \mathrm{~K}$ & $1200 \mathrm{k}$ & $1600 \mathrm{~K}$ \\
\hline$Q_{d}$ & $\sim 310^{11}$ & $\sim 3 \quad 10^{11}$ & $\sim 3 \quad 10^{12}$ & ح $310^{12}$ \\
\hline $\mathrm{Q}_{\mathbf{i}}$ & $\sim 110^{8}$ & $\sim 310^{7}$ & $\sim_{1} 10^{9}$ & $\sim 110^{9}$ \\
\hline
\end{tabular}

The indirect heating is always at least three orders of magnitude lower than the direct heating, and obviously negligible while the direct heating is present.

In between pulses the maximum possibie temperature rise due to indirect heating can be estimated from equation (30) by assuning the absence of conduction, so that

$$
\Delta \mathrm{T}_{\max }=\frac{K}{\mathrm{~K}} \mathrm{Q} \tau
$$

giving a worst possible temperature rise of .3 and 3 degrees Kelvin for aluminum and molybdenum, respectively. These are negligible since the samples car easily conduct away such small amounts of heat. 


\section{APPENDIX II \\ GREEN' ' FUNCTION METHOD FOR CALCULATING TEMPERATURE}

Equations

The two-dimensional time-dependent heat conduction equation in cylindrical coordinates is

$$
\frac{1}{K} \frac{\partial T}{\partial t}-\frac{1}{r} \frac{\partial}{\partial r}\left(r \frac{\partial T}{\partial r}\right)-\frac{\partial^{2} T}{\partial z^{2}}=\frac{1}{K} Q
$$

The region of interest is a finite cylinder of height $l$ and radius a silown in Fig. A-1, whose surface is held at a fixed temperature $T_{0}=T(t=0)$. Treating the right-hand side of equation (II-I) as a source term allows us to wite the formal solution in terms of the Green's function for the homogeneous equation [Carslaw and Jaeger, P. 380] as

$$
T(r, z, t)=\frac{K}{K} \int_{0}^{a} \int_{0}^{l} \int_{0}^{t} G\left(r, r^{\prime} ; z, z^{\prime} ; t, t^{-}\right) Q\left(r^{\prime}, z^{-}, t^{\prime}\right) 2 \pi r^{-} d r^{\prime} d z^{\prime} d t^{\prime}
$$

Tc find the necessary Green's function we use the method of separation of variables on the modified heat conduction equation

$$
\frac{1}{K} \frac{\partial G}{\partial t^{\prime}}-\frac{1}{r^{-}} \frac{\partial}{\partial r^{-}}\left(r^{-} \frac{\partial G}{\partial r}\right)-\frac{\partial^{2} G}{\partial z^{-2}}=0
$$

Assuming $G\left(r^{\prime}, z^{\prime}, t^{\prime}\right)=R\left(r^{\prime}\right) Z\left(z^{\prime}\right) \Theta\left(t^{\prime}\right)$ gives

$$
0=\frac{1}{K \Theta\left(t^{\prime}\right)} \frac{\partial \theta\left(t^{\prime}\right)}{\partial t^{\prime}}-\frac{1}{r^{\prime} R\left(r^{-}\right)} \frac{\partial}{\partial r^{\prime}}\left(r^{-} \frac{d R\left(r^{\prime}\right)}{d r^{\prime}}\right)-\frac{1}{Z\left(z^{\prime \prime}\right)} \frac{d^{2} z\left(z^{\prime}\right)}{d z^{-2}}
$$

which breaks up into the three ordinary differential equations

$$
\frac{d \Theta\left(t^{\prime}\right)}{d t^{\prime}}=-\left(c_{r}^{2}+c_{z}^{2}\right) k \theta\left(t^{\prime}\right)
$$




$$
\begin{aligned}
& \frac{d^{2} z\left(z^{\prime}\right)}{d z^{-2}}=-c_{z}^{2} z\left(z^{\prime}\right) \\
& \frac{1}{r^{\prime}} \frac{d}{d r^{\prime}}\left(r^{-} \frac{d R\left(r^{\prime}\right)}{d r^{\prime}}\right)=-c_{r}^{2} R\left(r^{\prime}\right)
\end{aligned}
$$

Equation (II-7) is the standard equation obeyed by Bessel function: ff order zero

$$
r^{* 2} \frac{d^{2} R}{d r^{* 2}}+r * \frac{d R}{d r *}+\left(r^{* 2}-(n=0)^{2}\right) R=0
$$

where $r^{*}=c_{r^{\prime}} r^{\prime}$. The boundary condition on this radial equation is

$$
T(a, z, t)=0 \text { (or a constant) }
$$

giving immediately $r^{\prime}=a \leftrightarrow r *=\alpha_{n}$ where $\alpha_{n}$ is the $n$th zero of $J_{0}\left(r^{*}\right)$, so that

$$
c_{r}=\frac{\alpha_{n}}{a}
$$

(The zeroeth order Bessel function of the second kind is eliminated since it cannot satisfy boundedness at $\mathbf{r}=0$.) The solution to the radial equation is then

$$
\mathrm{R}(\mathrm{r})=\sum_{\alpha_{n}} \mathrm{~A}_{\mathrm{n}} \mathrm{J}_{\mathrm{o}}\left(\alpha_{\mathrm{n}} \frac{\mathrm{r}^{\prime}}{\mathrm{a}}\right)
$$

where the coefficient $A_{n}$ is determined from the conditions

$$
R\left(r^{\prime}=r\right)=\delta\left(r^{\prime}-r\right)=f\left(r^{\prime}\right)
$$

to be 


$$
\begin{aligned}
& f\left(r^{\prime}\right)=\sum_{\alpha_{n}} A_{n} J_{0}\left(\alpha_{n} \frac{r^{\prime}}{a}\right) \\
& \int_{0}^{a} 2 \pi^{\prime} d r^{\prime} J_{0}\left(\alpha_{m} \frac{r^{\prime}}{a}\right) \delta\left(r^{\prime}-r\right)=\int_{0}^{a} 2 \pi r^{\prime} d r^{-} \sum_{\alpha_{n}} A_{n} J_{0}\left(\alpha_{n} \frac{r^{\prime}}{a}\right) J_{0}\left(\alpha_{m} \frac{r^{\prime}}{a}\right) \\
& J_{0}\left(\alpha_{m} \frac{r}{a}\right)=\pi a^{2} A_{m}\left[J_{1}\left(\alpha_{m}\right)\right]^{2} \\
& A_{m}=\frac{1}{\pi a^{2}} \frac{J_{0}\left(\alpha_{m} \frac{r}{a}\right)}{\left[J_{0}^{\prime}\left(\alpha_{m}\right)\right]^{2}}
\end{aligned}
$$

giving finally

$$
R\left(r, r^{\prime}\right)=\frac{I}{\pi a^{2}} \sum_{\alpha} \frac{J_{0}\left(\alpha_{n} \frac{r^{\prime}}{a}\right) J_{0}\left(\alpha_{n} \frac{r}{2}\right)}{\left[J_{0}^{\prime}\left(\alpha_{n}\right)\right]^{2}}
$$

Equation (II-6) is even simpler in that the solution are trigonometric rather than Bessel functions. Using the boundary condition

$$
Z\left(z^{\circ}=0\right)=Z\left(z^{\circ}=\ell\right)=0
$$

gives the solution

$$
\mathrm{Z}\left(\mathrm{z}^{\prime}\right)=\sum_{\mathrm{m}} B_{\mathrm{m}} \sin \left(\frac{\mathrm{m} \pi \mathrm{z}}{\mathrm{l}}\right)
$$

where the cosines disappear because of the $z=0$ condition, and the $z=\ell$ condition gives

$$
c_{z} l=m \pi \text { or } c_{z}=\frac{m \pi}{\ell}
$$

The coefficients $B_{m}$ are evaluated using 


$$
Z\left(z^{\circ}=z\right)=\delta\left(z-z^{\prime}\right)=f\left(z^{\prime}\right)
$$

to give.

$$
\begin{aligned}
& \int_{0}^{l} \sin \left(\frac{n \pi z}{l}\right) \delta\left(z-z^{\prime}\right) d z^{\prime}=\int_{0}^{l} \sum_{m} i_{n} \sin \left(\frac{n \pi z^{\prime}}{l}\right) \sin \left(\frac{n \pi z^{\prime}}{l}\right) d z^{-} \\
& \sin \left(\frac{n \pi z}{l}\right)=\frac{l}{2} A_{n} \\
& A_{n}=\frac{2}{l} \sin \frac{n \pi z}{l}
\end{aligned}
$$

giving fina11y

$$
Z\left(z, z^{\prime}\right)=\frac{2}{\ell} \sum_{\mathrm{m}} \sin \left(\frac{\mathrm{m} \pi \mathrm{z}^{\prime}}{\ell}\right) \sin \left(\frac{\mathrm{m} \pi \mathrm{z}}{\ell}\right)
$$

Utilizing the results of equations (II-10) and (II-17) allows equation (II-5) to be written as

$$
\frac{d \theta\left(t^{\prime}\right)}{d t^{\prime}}=-k\left[\frac{\alpha_{n}^{2}}{a^{2}}+\frac{(m \pi)^{2}}{l^{2}}\right] \theta\left(t^{\prime}\right)
$$

whose solution is

$$
\theta(t, t)=\sum_{\alpha} \sum_{n} \exp \left\{-k\left[\frac{\alpha_{n}^{2}}{a^{2}}+\frac{(m \pi)^{2}}{l^{2}}\right]\left(t-t^{\circ}\right)\right\}
$$

Thus the Green's function for this problem is

$$
\begin{gathered}
G\left(r, r^{\prime} ; z, z^{\prime} ; t, t^{\prime}\right)=\frac{2}{\pi a^{2} l} \sum_{m=1}^{\infty} \exp \left[-\kappa \frac{m^{2} \pi^{2}}{l^{2}}\left(t-t^{\prime}\right)\right] \sin \left(\frac{m \pi z}{l}\right) \sin \left(\frac{m \pi z^{\prime}}{l}\right) \\
\quad x \frac{\alpha_{n}}{\exp }\left[-\kappa \frac{\alpha_{n}^{2}}{a^{2}}\left(t-t^{-}\right)\right] \frac{J_{o}\left(\alpha_{n} \frac{r^{\prime}}{a}\right) J_{o}\left(\alpha_{n} \frac{r}{a}\right)}{\left[J_{o}^{\prime}\left(\alpha_{n}\right)\right]^{2}}
\end{gathered}
$$




\section{Application}

The LAMPF $800 \mathrm{MeV}$ pulsed proton beam has an elliptically-shaped target area approximated here by a circular area of radius a. Irradiation and heating of a thin rectangular foil whose edges and surface are held constant at the initial temperature can be approximated by considering heat conduction and generation in a finite cylinder large enough that the radial edges see little irradiation. The proton flux for the LAMPF beam is Gaussian in profile and hence can be described as

$$
I(r, z, t)=I(t) \exp \left(-\frac{r^{2}}{r_{0}^{2}}\right)
$$

where $r_{0}$ is a constant $(=\sqrt{2 \sigma}$ where $\sigma$ is the standard deviation of the Gaussian profile) giving the average size of the beam spot and $I(t)$ is a pulsed time function equal to

$$
I(t)=\left\{\begin{array}{l}
0 \text { for } t<0 \\
I_{0} \text { for } m \tau \leq t \leq m \tau+\tau_{1} \\
0 \text { for } m \tau+\tau_{1} \leq t \leq(m+1) \tau
\end{array}\right.
$$

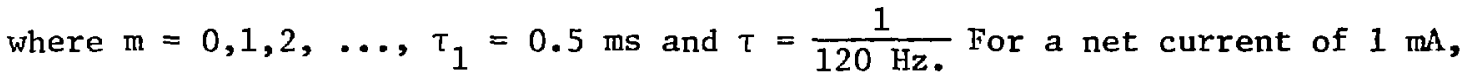
$I_{0}$ is given by

$$
I_{C}=\frac{n_{p} / \tau_{1}}{\int_{0}^{a} 2 \pi r \exp \left(-\frac{r^{2}}{r_{0}^{2}}\right) d r} \stackrel{\simeq \frac{n_{p} / \tau_{1}}{\pi r_{0}^{2}}}{=}
$$

where $n_{p}$ is the total number of protons in a pulse occurring every $T$ seconds, given by

$$
\mathrm{n}_{\mathrm{p}}=1 \mathrm{~mA} \times \frac{10^{-3} \mathrm{C}}{\mathrm{sec}-\mathrm{mA}} \times 6.2810^{18} \frac{\mathrm{P}}{\mathrm{C}} \times \frac{1}{120 \mathrm{pulse} / \mathrm{sec}}=5.23310^{13} \frac{\mathrm{p}}{\mathrm{pu} 1 \mathrm{se}}
$$

for a net current of $1 \mathrm{~mA}$ with 120 pulses per second. If each $300 \mathrm{MeV}$ proton loses an amount of energy $\Xi^{*}$ per unit distance traversed in the target, and if all this energy is assumed to produce heat by direct excitation of the lattice, 
the heat generation rate per unit volume is

$$
Q=E^{*} \frac{n_{p} / \tau_{1}}{\pi r_{0}^{2}} \exp \left(-\frac{r^{2}}{r_{0}^{2}}\right)=Q_{0} \exp \left(-\frac{r^{2}}{r_{0}^{2}}\right)=c_{Q_{0}} I_{0} \exp \left(-\frac{r^{2}}{r_{0}^{2}}\right)
$$

whenever the bealn is on, and zero otherwise. To insure that most of the beam is accounted for we set $a=3 \sigma=2.12132 r_{0}$ (by which time we are using $99 \%$ of the current).

The temperature is given by equations (2) and (23) as

$$
\begin{aligned}
& T(r, z, t)-T_{0}=\frac{\kappa}{K} a_{0} \frac{2}{\pi a^{2} l} \sum_{m=1}^{\infty} \sum_{\alpha, ~} \int_{0}^{=} \exp \left\{-\kappa\left[\frac{m^{2} \pi^{2}}{l^{2}}+\frac{\alpha_{n}^{2}}{a^{2}}\right]\left(t-t^{\prime}\right)\right\} f\left(t^{\prime}\right) d^{-} \\
& x \int_{0}^{a} 2 \pi^{-} \exp \left(-\frac{r^{-2}}{r_{0}^{2}}\right) \frac{J_{0}\left(\alpha_{n a}\right) J_{0}\left(\alpha_{n} \frac{r^{\prime}}{a}\right)}{\left[J_{0}^{\prime}\left(\alpha_{n}\right)\right]^{2}} d r^{\prime} \int_{0}^{l} \sin \left(\frac{m \pi z}{l}\right) \sin \left(\frac{m \pi z^{\prime}}{l}\right) d_{z^{\prime}}
\end{aligned}
$$

where $f\left(t^{\prime}\right)$ is a pulsed time function such as given by equation (25) but normalized to unity.

The integration in $z^{\prime}$ is readily carried out to give

$$
\int_{0}^{\ell} \sin \left(\frac{m \pi z}{\ell}\right) \sin \left(\frac{m \pi z^{-}}{\ell}\right) d z^{-}=\frac{2 \ell}{m \pi} \sin \left(\frac{m \pi z}{\ell}\right) m=1,3,5, \ldots
$$

and the summation over odd $m$ can be rewritten as $m \rightarrow 2 m+1$. The integration in $\mathrm{r}^{-}$can only be approximated, using the definite integral

$$
\int_{0}^{\infty} r^{v+1} e^{-a^{2} r^{2}} J_{v}(b r) d r=\frac{b^{v}}{(2 a)^{v+1}} \exp \left(-\frac{b^{2}}{4 a^{2}}\right)
$$

to give

$$
\int_{0}^{a} 2 \pi^{-} \frac{J_{0}\left(\alpha_{n} \frac{r^{\prime}}{a}\right) J_{0}\left(\alpha_{n} \frac{r}{a}\right)}{\left[J_{0}^{\prime}\left(\alpha_{n}\right)\right]^{2}} \exp \left[-\frac{r^{-2}}{r_{0}^{2}}\right] d r^{-\cong} \cong 2 \frac{J_{0}\left(\alpha_{n} \frac{r}{a}\right)}{J_{1}{ }^{2}\left(\alpha_{n}\right)} \sigma^{2} \exp \left[-\frac{\alpha_{n}^{2}}{18}\right]
$$




$$
\cong \pi r_{0}^{2} \frac{J_{0}\left(\alpha_{n} \frac{r}{n}\right)}{J_{l}^{2}\left(\alpha_{n}\right)} \exp \left(-\frac{\alpha_{n}^{2}}{18}\right)
$$

where we have used the relations $J_{0}{ }^{-}\left(\alpha_{i}\right)=J_{1}\left(\alpha_{n}\right)$ and $2 \sigma^{2}=r_{0}^{2}=\frac{a^{2}}{4.5}$.

Due to the rapidly decaying nature of the Gaussian the approximation a $\pi^{\infty}$ in the integration limit is quite reasonable.

The integration in $t^{\prime}$ is done by defining the integral

$$
I_{\alpha m}=\beta_{\alpha m} \int_{-1}^{t} \exp \left(\beta_{\alpha m} t^{\prime}\right) f\left(t^{\prime}\right) d t^{\prime}
$$

where

$$
\beta_{\alpha m}=\left(\frac{\alpha^{2}}{a^{2}}+\frac{(2 m+1)^{2} \pi^{2}}{l^{2}}\right) \kappa
$$

Since $f\left(t^{\prime}\right)$ only exists for $k \tau \leq t \leq k \tau+\tau_{1}$ the integral $I_{\alpha \mathrm{m}}$ reduces to

$$
\begin{aligned}
& I_{\alpha m}=\beta_{\alpha m} \int_{0}^{\tau} \exp \left(\beta_{\alpha m^{\prime}} t^{\prime}\right) d t^{-}+\int_{\tau}^{\tau+\tau} 1 \quad \beta_{\alpha m} \exp \left(\beta_{\alpha m} t^{\prime}\right) d t+\ldots+ \\
& +\beta_{\alpha m} \int_{(k-1) \tau}^{(k-1) \tau+\tau} 1 \exp \left(\beta_{\alpha m^{\prime}}\right) d t^{\prime} \\
& +\int_{k \tau}^{t} \text { or }{ }^{k \tau+\tau} 1 \beta_{\alpha m} \exp \left(\beta_{\alpha m} t^{-}\right) d t^{\prime}
\end{aligned}
$$

The lower limits give the sum

$$
-1-\exp \left(\beta_{\alpha \mathrm{m}} \tau\right)-\exp \left(2 \beta_{\alpha \mathrm{m}} \tau\right)-\ldots-\exp \left(k \beta_{\alpha \mathrm{m}} \tau\right)=\frac{\exp \left[(k+1) \beta_{\alpha m} \tau\right]-1}{I-\exp \left(\beta_{\alpha \mathrm{m}} \tau\right)}
$$

while the upper limits give 


$$
\begin{aligned}
& \exp \left(\beta_{\alpha \mathrm{m}} \tau_{1}\right)\left\{1+\exp \left(\beta_{\alpha \mathrm{m}} \tau\right)\right. \\
& \left.\left.+\exp \left(\beta_{\alpha \mathrm{m}} 2 \tau\right)+\ldots+\exp \left[(k-1) \beta_{\alpha \mathrm{m}} \tau\right)\right]\right\} \\
& +\left\{\begin{array}{l}
\exp \left(\beta_{\alpha \mathrm{m}} \tau\right) \\
\exp \left[\beta_{\alpha \mathrm{m}}\left(k \tau+\tau_{1}\right)\right]
\end{array}\right. \\
& =\exp \left(\beta_{\alpha \mathrm{m}} \tau_{1}\right)\left[\frac{\exp \left(k \beta_{\alpha \mathrm{m}} \tau\right)-1}{\exp \left(\beta_{\alpha \mathrm{m}} \tau\right)-1}\right]+\left\{\begin{array}{l}
\exp \left(\beta_{\alpha \mathrm{m}} \tau\right) \\
\exp \left[\beta_{\alpha \mathrm{m}}\left(k \tau+\tau_{1}\right)\right]
\end{array}\right.
\end{aligned}
$$

Combining these results yields

$$
\begin{aligned}
I_{\alpha \mathrm{m}}=\frac{1-\exp \left[(k+1) \beta_{\alpha \mathrm{m}} \tau\right]+\exp \left[\beta_{\alpha_{\mathrm{m}}}\left(k \tau+\tau_{1}\right)\right]-\exp \left(\beta_{\alpha \mathrm{m}} \tau_{1}\right)}{\exp \left(\beta_{\alpha \mathrm{m}} \tau\right)-1} \\
+\int_{\exp \left[\beta_{\alpha \mathrm{m}}\left(k \tau+\tau_{1}\right)\right]}^{\exp \left(\beta_{\alpha \mathrm{m}} \tau\right)}
\end{aligned}
$$

where the two final terms refer respectively to times dirin's and between pulses. Putting together equations (II-29), (II-30), (II-32) and (II-38) gives as the entire solution

$$
\begin{aligned}
& T(r, z, t)-T_{0}=\frac{K}{K} Q_{0} \frac{2}{\pi a^{2} \ell} \sum_{m}\left[\frac{2 \ell}{(2 m+1) \pi} \sin \left(\frac{(2 m+1) \pi z}{\ell}\right)\right] \\
& \times \sum_{\alpha} \pi r_{0}^{2} \exp \left(-\frac{\alpha_{n}^{2}}{18}\right) \frac{J_{n}\left(\alpha_{n} \frac{r}{a}\right)}{J_{1}^{2}\left(\alpha_{n}\right)} \times \frac{1}{\kappa}\left(\frac{a^{2} \ell^{2}}{\alpha_{n}^{2} n^{2}+(2 m+1)^{2} \pi^{2} a^{2}}\right) \\
& x \exp \left(-\beta_{\alpha m} t\right)\left\{\frac{1-\exp \left[(k+1) \beta_{\alpha m} \tau\right]+\exp \left[\beta_{\alpha m}\left(k \tau+\tau_{1}\right)\right]-\exp \left(\beta_{\alpha m} \tau_{1}\right)}{\exp \left(\beta_{\alpha m} \tau\right)-1}\right. \\
& +\exp \left(\beta_{\alpha m} t\right)
\end{aligned}
$$




$$
\begin{aligned}
& T(r, z, t)-T_{0}=\frac{\kappa}{K} Q_{,} \frac{2}{\pi a^{2} \ell} \sum_{m} \frac{2 \ell}{(2 m+1) \pi} \sin \frac{(2 m+1) \pi z}{\ell} \\
& x \sum_{\alpha} \pi r_{n}^{2} \exp \left(-\frac{\alpha_{n}^{2}}{18}\right) \frac{J_{0}\left(\alpha_{n} \frac{r}{a}\right)}{J_{1}^{2}\left(\alpha_{n}\right)} \times \frac{1}{k}\left(\frac{a^{2} \ell^{2}}{\alpha_{n}^{2} \ell^{2}+(2 m+1)^{2} \pi^{2} a^{2}}\right) \\
& \quad x \exp \left(-\beta_{\alpha m} t\right)\left\{\frac{1-\exp \left[(k+1) \beta_{\alpha m} \tau\right]+\exp \left[\beta_{\alpha m}\left(k \tau+\tau_{1}\right)\right]-\exp \left(\beta_{\alpha m} \tau_{1}\right)}{\exp \left(\beta_{\alpha m} \tau\right)-1}\right. \\
& \left.+\exp \left[\beta_{\alpha m}\left(k \tau+\tau_{1}\right)\right]\right\}
\end{aligned}
$$$$
\text { for } k \tau+\tau, \leq t \leq(k+1) \tau
$$

Rearranging the constants and returning to ine $I_{\alpha m}$ notation gives

$$
\begin{aligned}
T(x, z, t) & =\mathrm{T}_{0}+ \\
& \frac{\mathrm{Q}}{\mathrm{K}} \frac{8}{9 \pi} \sum_{\mathrm{m}} \frac{\sin \left(\frac{(2 \mathrm{~m}+1) \pi \mathrm{z}}{l}\right)}{(2 \mathrm{~m}+1)} \sum_{\alpha_{\mathrm{n}}} \exp \left(-\frac{\alpha_{\mathrm{n}}{ }^{2}}{18}\right) \frac{\mathrm{J}_{\mathrm{j}}\left(\alpha_{\mathrm{n}} \frac{\mathrm{r}}{\mathrm{a}}\right)}{\mathrm{J}_{1}^{2}\left(\alpha_{\mathrm{n}}\right)} \frac{\kappa}{\beta_{\alpha \mathrm{m}}} \exp \left(-\beta_{\alpha \mathrm{m}}{ }^{\mathrm{t}}\right) \mathrm{I}_{\alpha \mathrm{m}}
\end{aligned}
$$

Results

Two limiting cases of equation (II-39) are of particular interest. The sample may be constrained to return to its initial temperature $T_{0}$ throughout before the arrival of the next heating pulse, resulting in perfectly periodic and identical temperature oscillations (studied by setting $k=0$ ). If all the heat deposited in the sample cannot be conducted away before the arrival of the next pulse on the other hand, the temperature will rise in a stepwise fashion until at some possibly much higher average temperature the conduction losses balance the irradiation heating, and the temperature oscillations are again periodic and identical (studied by setting $k=\infty$ ). This case is of interest since the final temperatures may be above the sample melting temperature. Only the first is considered here. 
In a controlled-temperature experiment the maximum temperature rise (occurring at the end of each pulse $t=\tau_{1}$ and at the sample midpoint $r=0$ and $z=\frac{l}{2}$ ) would be limited to a small fraction of the melting temperature, and the sample would ideally return to its initial state before the arrival of the next heating pulse. The behavior of the temperature during and immediately after the first $(k=0)$ pulse would then describe the behavior for all subsequent times. The temperature rise at the sample center $\left(r=0\right.$ and $\left.z=\frac{l}{2}\right)$ is given by

$$
\begin{aligned}
& T\left(0, \frac{\ell}{2} t\right)=T_{0}+\frac{Q}{k}-\frac{8}{j \pi} \sum_{m} \frac{(-1)^{m}}{(2 m+1)} \sum_{\alpha_{n}} \frac{\exp \left(\frac{-\alpha_{n}^{2}}{18}\right)}{J_{1}^{2}\left(\alpha_{n}\right)}\left(\frac{a^{2} \ell^{2}}{\alpha_{n}^{2} \ell^{2}+(2 m+1)^{2} \pi^{2} a^{2}}\right) \\
& x\left[1-\exp \left(-\beta_{\alpha m} t\right)\right] \\
& \text { for } 0 \leq t \leq \tau_{1} \\
& \mathrm{~T}\left(0, \frac{\ell}{2}, \mathrm{t}\right)=\mathrm{T}_{0}+\frac{Q}{\mathrm{k}} \frac{8}{9 \pi} \sum_{m} \frac{(-1)^{m}}{(2 m+1)} \sum_{\alpha_{n}} \frac{\exp \left(\frac{-\alpha_{n}^{2}}{13}\right)}{J_{1}^{2}\left(\alpha_{n}\right)}\left(\frac{a^{2} \ell^{2}}{\alpha_{n}^{2} \ell^{2}+(2 \pi+1)^{2} \pi^{2} a^{2}}\right) \\
& x \exp \left(-\beta_{\alpha m} t\right)\left[\exp \left(B_{c m} \tau_{1}\right)-1\right] \\
& \text { for } \tau_{1} \leq t \leq \tau
\end{aligned}
$$

The maximum centerline temperature rise (occuring at $t=\tau_{1}$ ) is shown for aluminum in figure $A-2$ and molybdenum in figure $A-3$, for various values of beam current, Gaussian width and sample thickness. The centerline temperature at the beginning of the next heating pulse $(t=\tau)$ is shown in figures $A-4$ and $A-5$, for aluminum and molybdenum respectively.

The maximum temperature rise occurs at the center of the sample $\quad r=0$ and $z=\frac{l}{2}$ ) and the temperature then falls of $f$ both radially and axially to the fixed surface temperature $T_{0}$. The central temperature is shown in figure $A-6$ as a function of time for aiuminum, using the parameters given in tables 1 and 2 . 
The radial profiles at $z=\frac{\ell}{2}$ and the axial profiles at $r=0$ are shown in $f 1 g-$ ures $A-7$ and $A-8$ for several different times. Figures $A-9$, and A-10 and A-11 give the same results for molybdenun, using, the parameters given in tables 1 and 2 .

The temperature solution developed is applicable to an arbitrary beam time structure (varying $\tau_{1}$ and $\tau$ ). The effect of changing $\tau_{1}$ is shown in figures $A-12$ and $A-13$, where the centerline temperature rise in aluminum and molybdenum is shown as a function of time for various values of $\tau_{1}$, for the same parameters used above. The maximum temperature rise (given in table 3), occurring at $t=\tau_{1}$, increases as the heating pulse becomes shorter and more intense (the net amount of heat deposited is held fixed). A limiting value of tinis maximam temperature rise is reached when essentially none of the heat can be conducted away during the pulse itself, and is given by

$$
\frac{\tau_{1}{ }^{\lim }}{\tau} \rightarrow 0 \Delta T_{\max }=\frac{\kappa}{k} c_{Q} \frac{n_{p}}{\pi r_{0}^{2}}
$$

The temperature rise at $t=\tau_{1}=10^{-3}$ T has indeed reached this limiting value. 
TABLE：

Assumptions and Constants

Dislocation Density

Void Number Density

Interstitial Loop Number Density

Intial Vacancy Loop Number Dens:ty

Initial Void Radius

Initial Interstitial Loop Radius

Vacancy Loop Formation Radius

Gas Atoms per Void

Van der Waal's Coefficient

Boltzmann's Constant

Vacancy Frequency of Motion

Interstitial Frequency of Motion

Combinatory Number $\rho_{d}^{0}$

$N_{v}$

$N_{\text {il }}$

$\mathrm{v}_{\mathrm{v} \mathrm{Q}^{(0)}}$

$r_{v}(0)$

$r_{i \ell}(0)$

$r_{\mathrm{v} \ell}(0)$

$\mathrm{n}_{\mathrm{g}}$

bv

b

$v_{v}$

$v_{1}$

g

$$
10^{11}
$$$$
10^{20}
$$$$
10^{21}
$$

0

$10^{-3}$

$2.410^{-8}$

$1.510^{-9}$

10

$8.310^{-29}$

$n^{3}$

$1.3810^{-23} \mathrm{~J} /{ }^{\circ} \mathrm{K}$

$10^{13}$

$s^{-1}$

$10^{13}$

$s^{-1}$ $\mathrm{m} / \mathrm{n}^{3}$

$m^{-3}$

$\mathrm{n}^{-3}$

$m^{-3}$

in

m

m 
TABLE 2

Material Properties

\begin{tabular}{|c|c|c|c|c|}
\hline & & Aluminum & Molybdenum & Units \\
\hline Burgers vector & b & $2.3210^{-10}$ & $2.7310^{-10}$ & $\mathrm{~m}$ \\
\hline Vacancy formation energy & $\mathrm{E}_{\mathrm{f}}^{\mathrm{V}}$ & $1.0610^{-19}$ & $4.8 \quad 10^{-13}$ & $J$ \\
\hline Interstitial formation energy & $\mathrm{E}_{\mathrm{f}}^{\dot{i}}$ & $5.1910^{-19}$ & $1.4410^{-18}$ & $\mathbf{J}$ \\
\hline Vacancy migration eisergy & $\mathrm{E}_{\mathrm{m}}^{\mathrm{v}}$ & $9.1210^{-20}$ & $2.4510^{-19}$ & $\mathrm{~J}$ \\
\hline Interstitial migration energy & $\mathrm{E}_{\mathrm{m}}^{1}$ & $1.6 \quad 10^{-20}$ & $1.3610^{-20}$ & $\mathrm{~J}$ \\
\hline Vacancy diffusion pre-exponential & $\mathrm{D}_{\mathrm{v}}^{0}$ & $4.0 \quad 10^{-6}$ & $3.0 \quad 10^{-5}$ & $\mathrm{~m}^{2} / \mathrm{s}$ \\
\hline $\begin{array}{l}\text { Interstitial diffusion pre- } \\
\text { exponential }\end{array}$ & $D_{i}^{o}$ & $8.010^{-6}$ & $1.610^{-5}$ & $\mathrm{~m}^{2} / \mathrm{s}$ \\
\hline Shear modulus & $\mu$ & $2.4310^{10}$ & $1.1510^{11}$ & $\mathbf{P a}$ \\
\hline Polsson's ratio & $\nu$ & 0.347 & 0.32 & - \\
\hline Stacking fäult energy & $\gamma_{\text {sf }}$ & 0.28 & 0.574 & $\mathrm{~J} / \mathrm{m}^{2}$ \\
\hline Surface energy & $\gamma$ & 1.0 & 2.05 & $\mathrm{~J} / \mathrm{m}^{2}$ \\
\hline Cascade efficiency & $\varepsilon$ & 0.001 & 0.001 & - \\
\hline Vacancy dislocation bias factor & $\mathrm{z}_{\mathrm{v}}$ & 1.0 & 1.0 & - \\
\hline $\begin{array}{l}\text { Interstitial dislocation } \\
\text { bias factor }\end{array}$ & $z_{i}$ & 1.08 & 1.08 & - \\
\hline Thermal conductivity & $\mathrm{k}$ & 222.0 & 142.0 & $\mathrm{~J} / \mathrm{ms}^{\circ} \mathrm{K}$ \\
\hline Thermal diffusivity & K & $9.1310^{-5}$ & $5.04 \quad 10^{-5}$ & $\mathrm{~m}^{2} / \mathrm{s}$ \\
\hline Damage energy cross section & $\sigma_{\mathrm{D}}$ & 63.0 & 731.0 & $b-k e v$ \\
\hline Threshold energy & $\mathrm{E}_{\mathrm{D}}$ & 17.0 & 40.0 & ev \\
\hline $800 \mathrm{MeV}$ proton energy loss & E* & $8.0 \quad 10^{-11}$ & $2.4710^{-10}$ & $\mathrm{~J} / \mathrm{mp}$ \\
\hline Current & I & 1 & 0.5 & $\mathbf{m A}$ \\
\hline Gaussian size & $r_{0}$ & $3.110^{-3}$ & $1.18 \quad 10^{-3}$ & m \\
\hline Sample thickness & $\ell$ & $2.510^{-4}$ & $2.510^{-4}$ & $\mathbf{n}$ \\
\hline
\end{tabular}


TABLE 3

Radiation-produced Temperature Rises

\begin{tabular}{|c|c|c|}
\hline \multirow[t]{8}{*}{ Aluminum: } & $f^{\prime}(\%)$ & $\Delta T_{\max }$ \\
\hline & 100 & 0.58 \\
\hline & 50 & 1.17 \\
\hline & 25 & 2.33 \\
\hline & 10 & 5.84 \\
\hline & 6 & 9.72 \\
\hline & 1 & 40.28 \\
\hline & 0.1 & 56.94 \\
\hline \multirow[t]{8}{*}{ Molybdenum: } & $f(\%)$ & $\Delta \mathrm{T}_{\max }$ \\
\hline & 100 & 9.70 \\
\hline & 50 & 19.39 \\
\hline & 25 & 38.78 \\
\hline & 10 & 96.83 \\
\hline & 6 & 158.58 \\
\hline & 1 & 457.12 \\
\hline & 0.1 & 526.71 \\
\hline
\end{tabular}




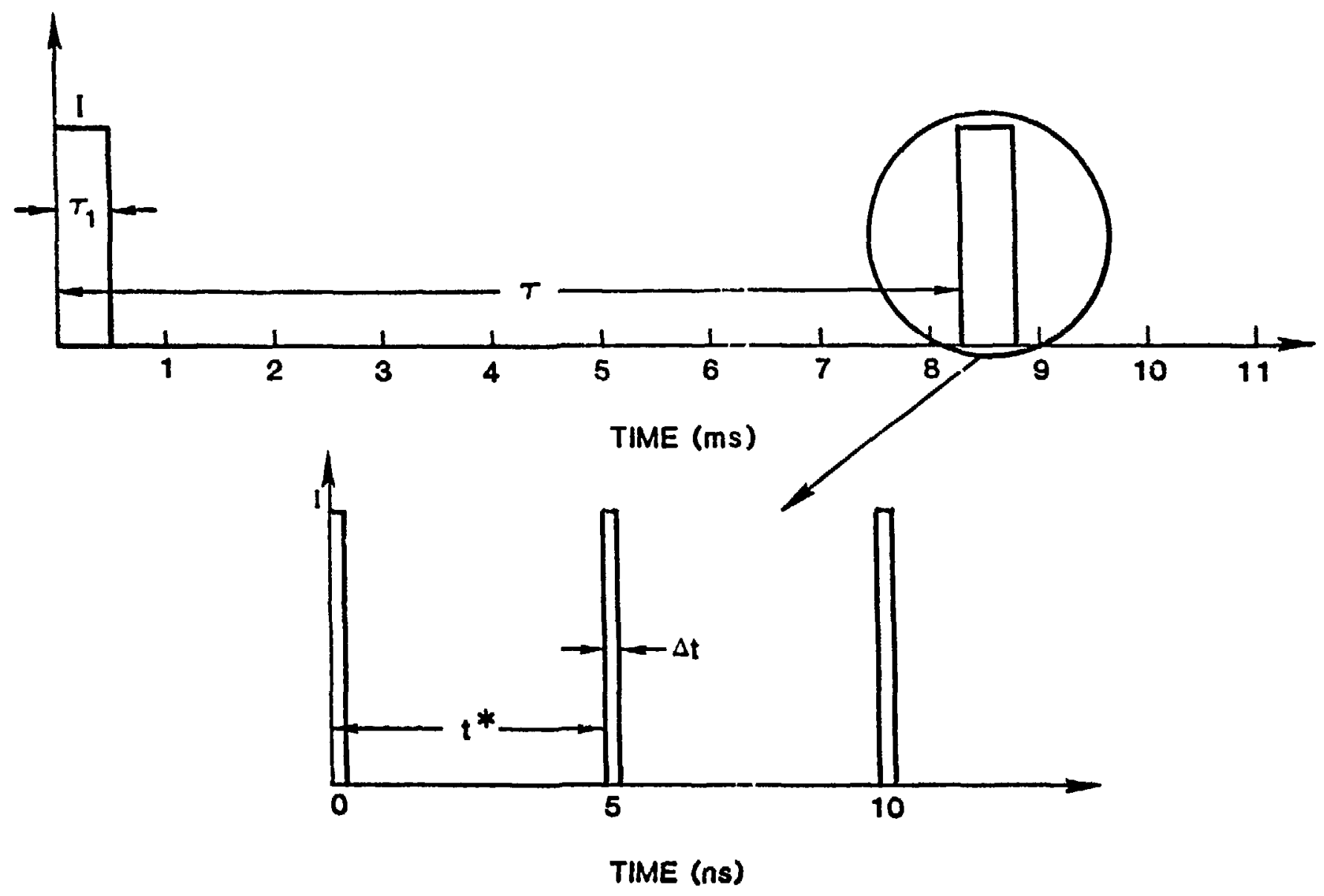

Figure 1. Time structure of LAMPF $800 \mathrm{MeV}$ proton beam. 


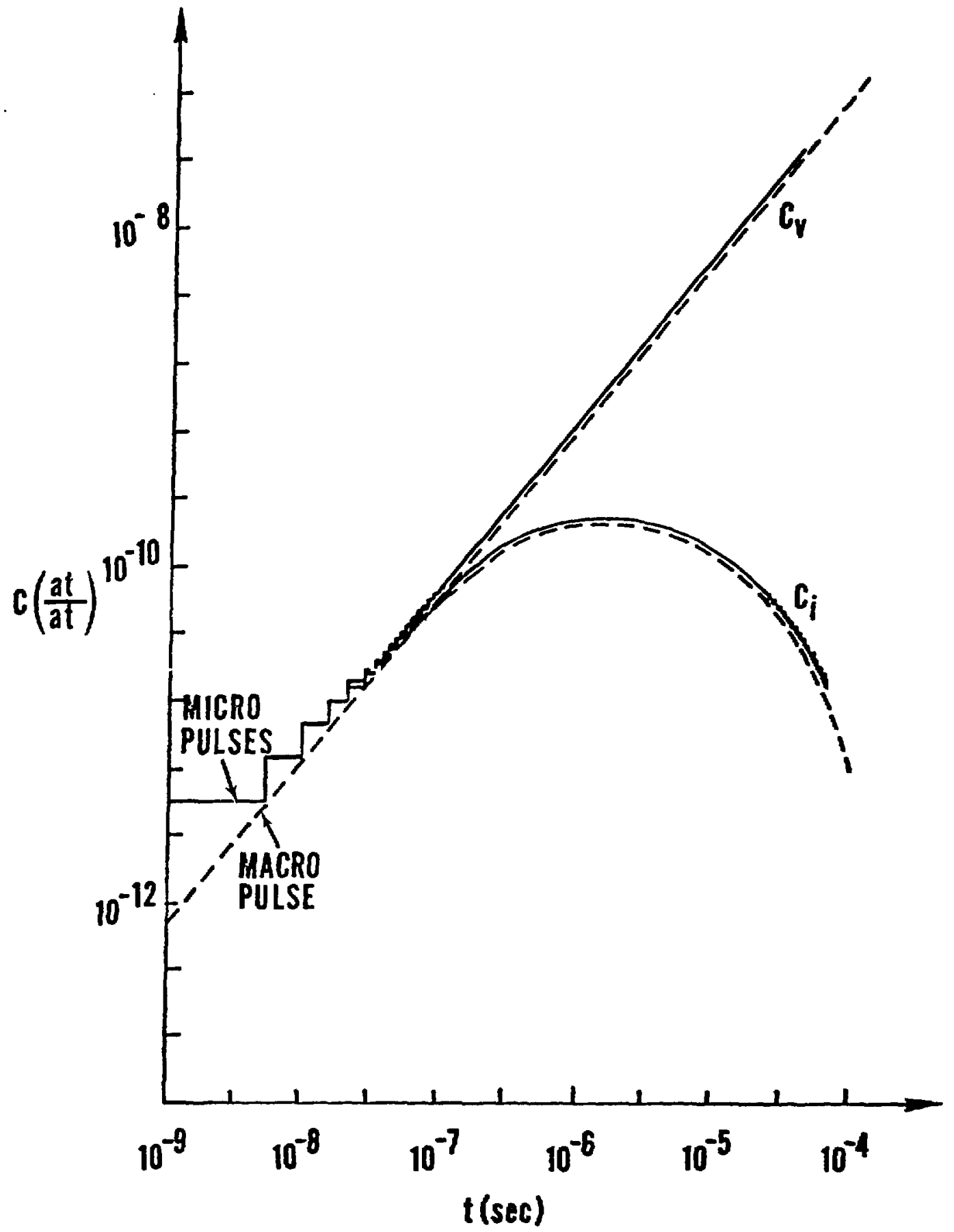

Figure 2. Vacancy and interstitial concentrations during first macropulse. 


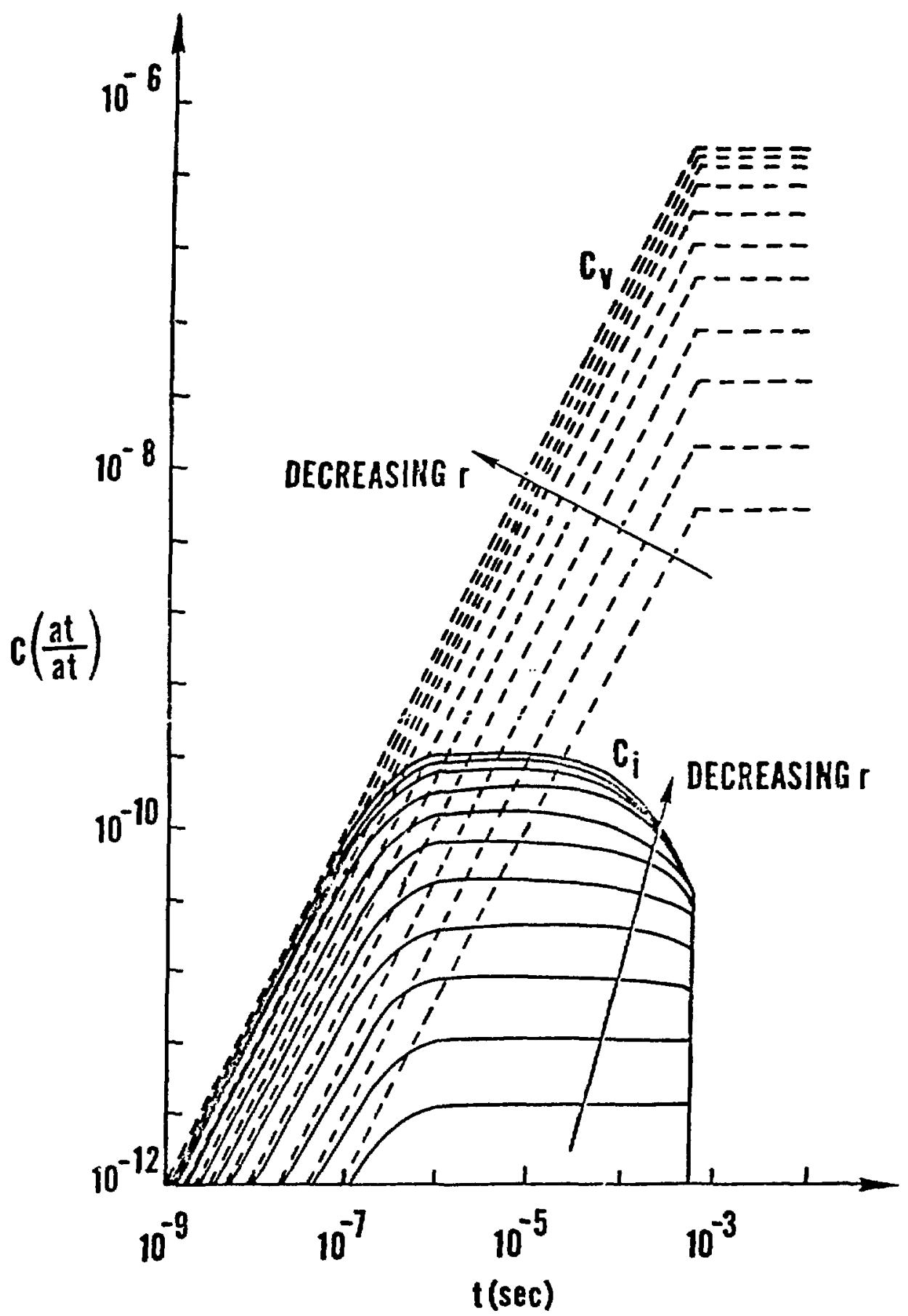
Figure 3 . Vacancy and interstitial concentrations during first pulse and
annealing period. For each set of curves, the upper curve corresponds to the center $(r=0$ of Fig. Al) of the beam and the lower curve to the edge $(r=3 \sigma$ of Fig. Al) of the beam. The intermediate curves correspond to equispaced positions from center to edge of the beam. 


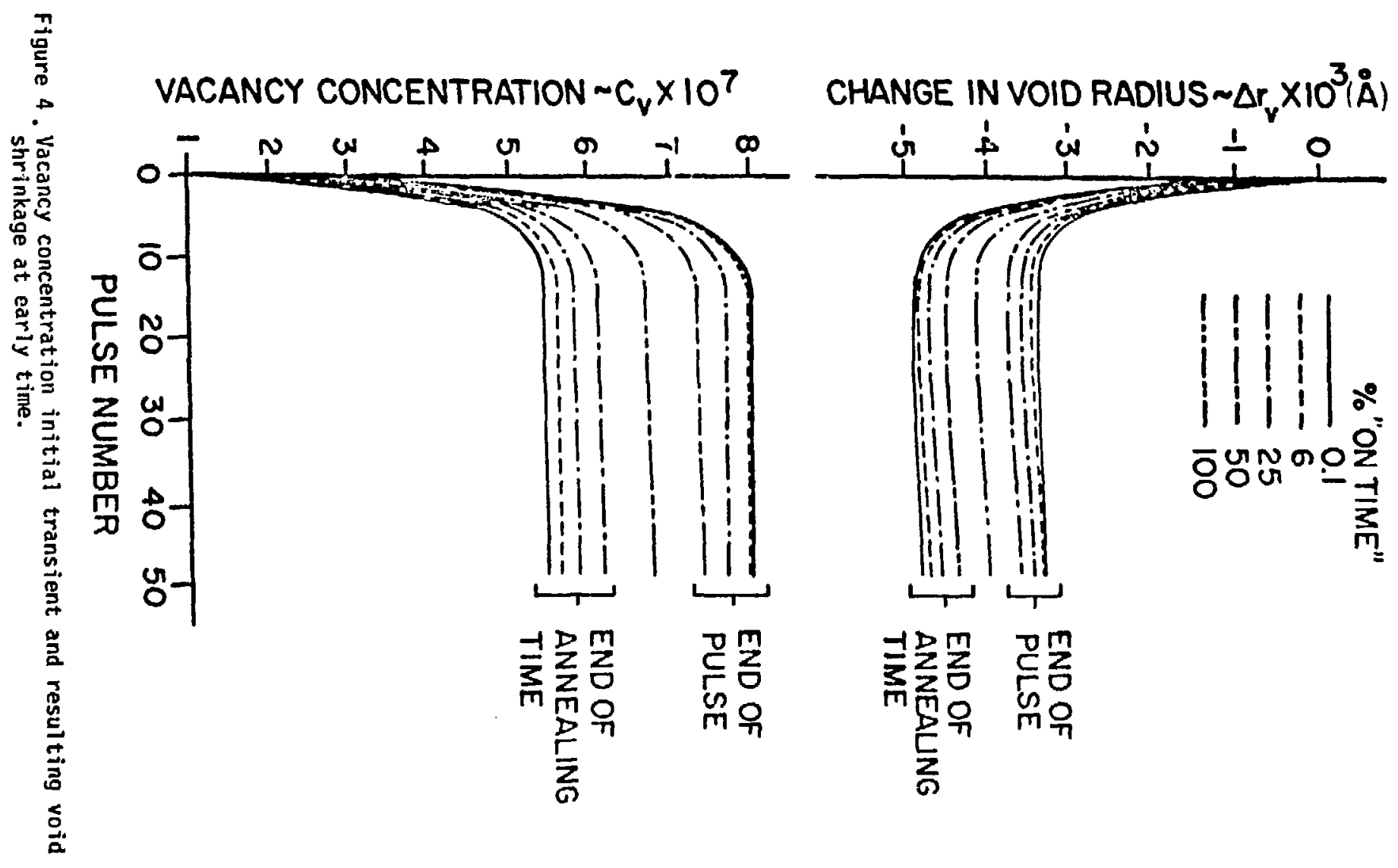




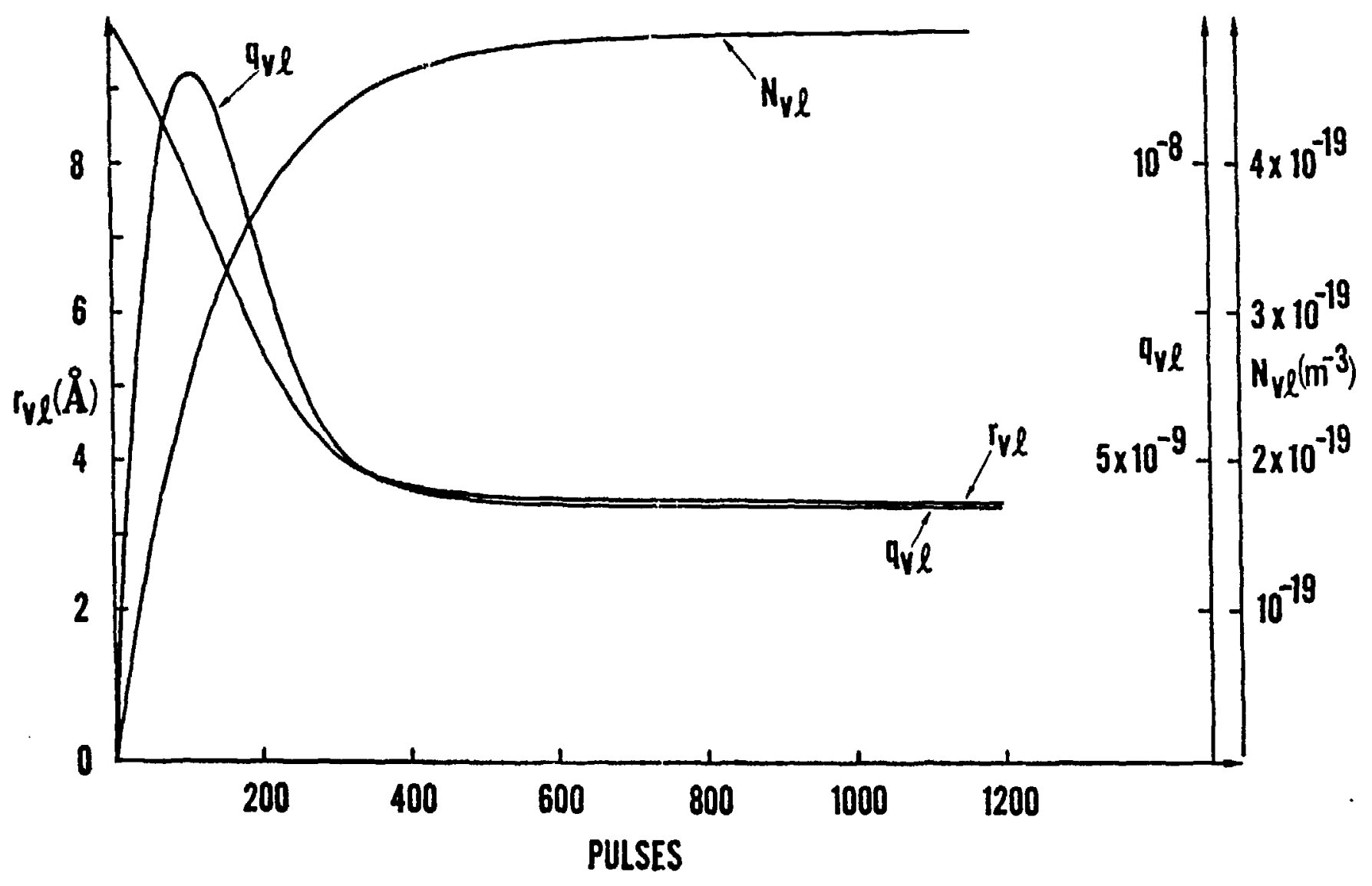

Figure 5. Vacancy loop size and number density transient. 


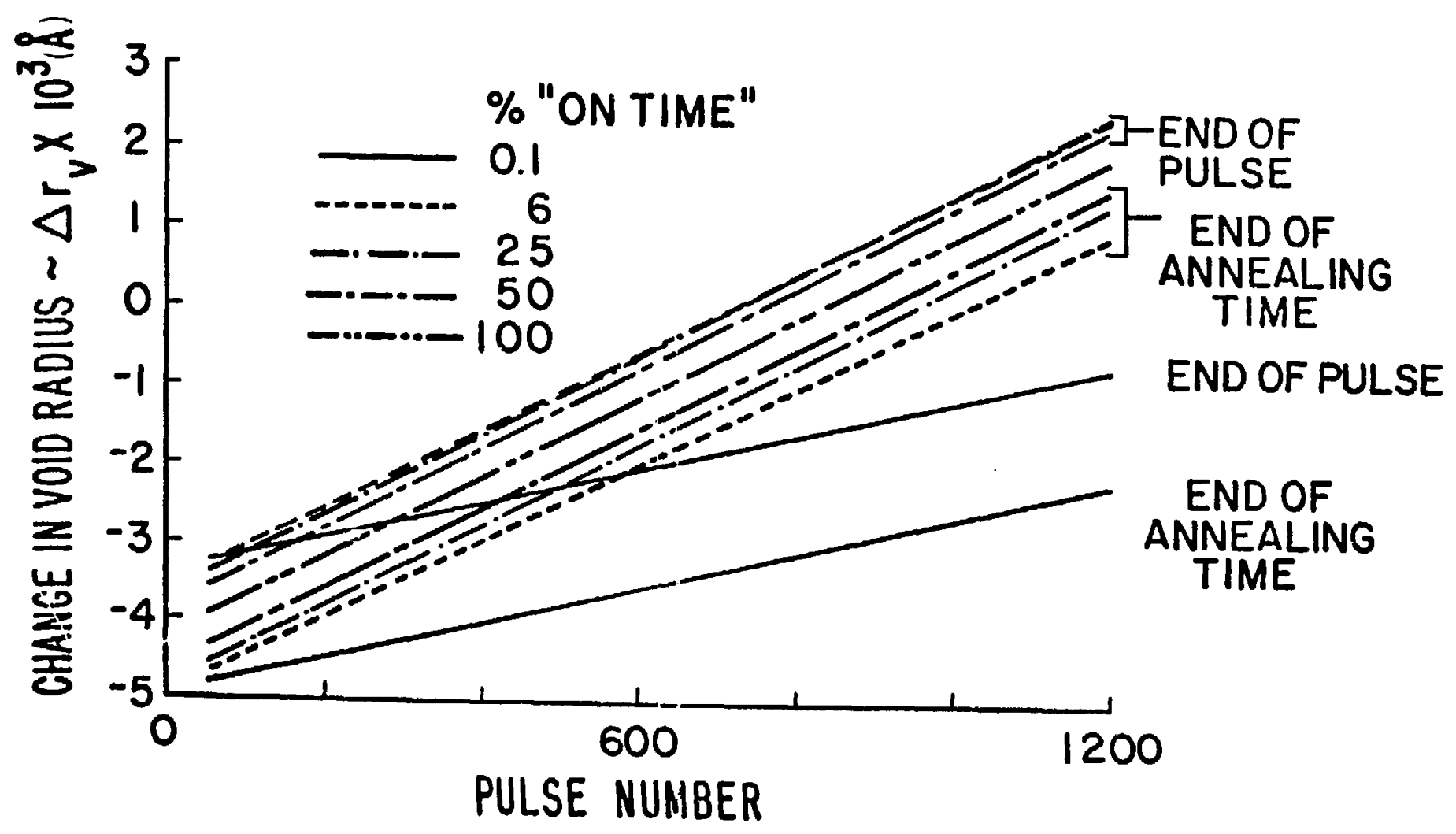

Figure 6. Void growth for various irradiations in aluminum at an ambient temperature of $400 \mathrm{~K}$. 


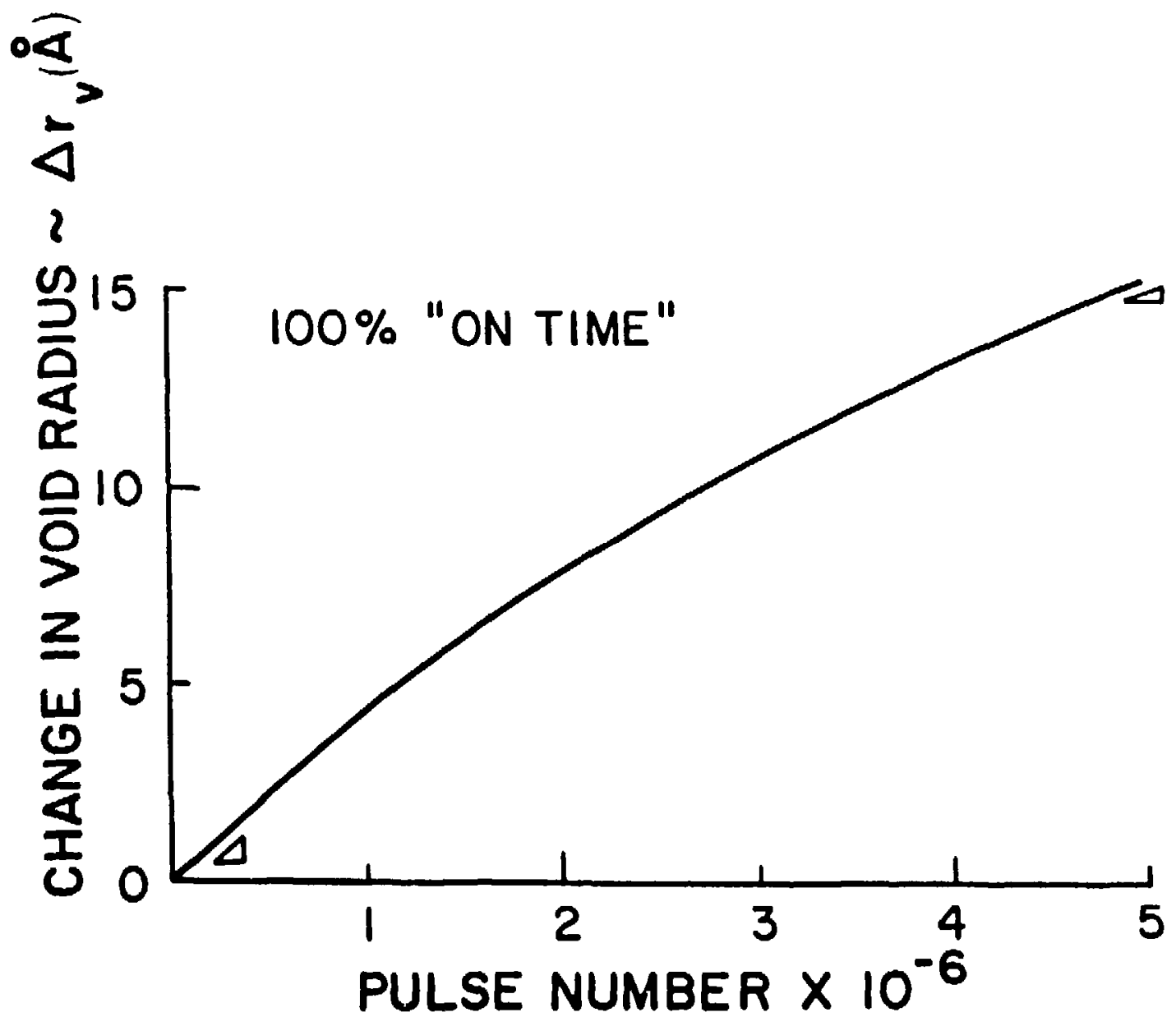

Figure 7. Long-term void growth in aluninum at $400 \mathrm{~K}$. 


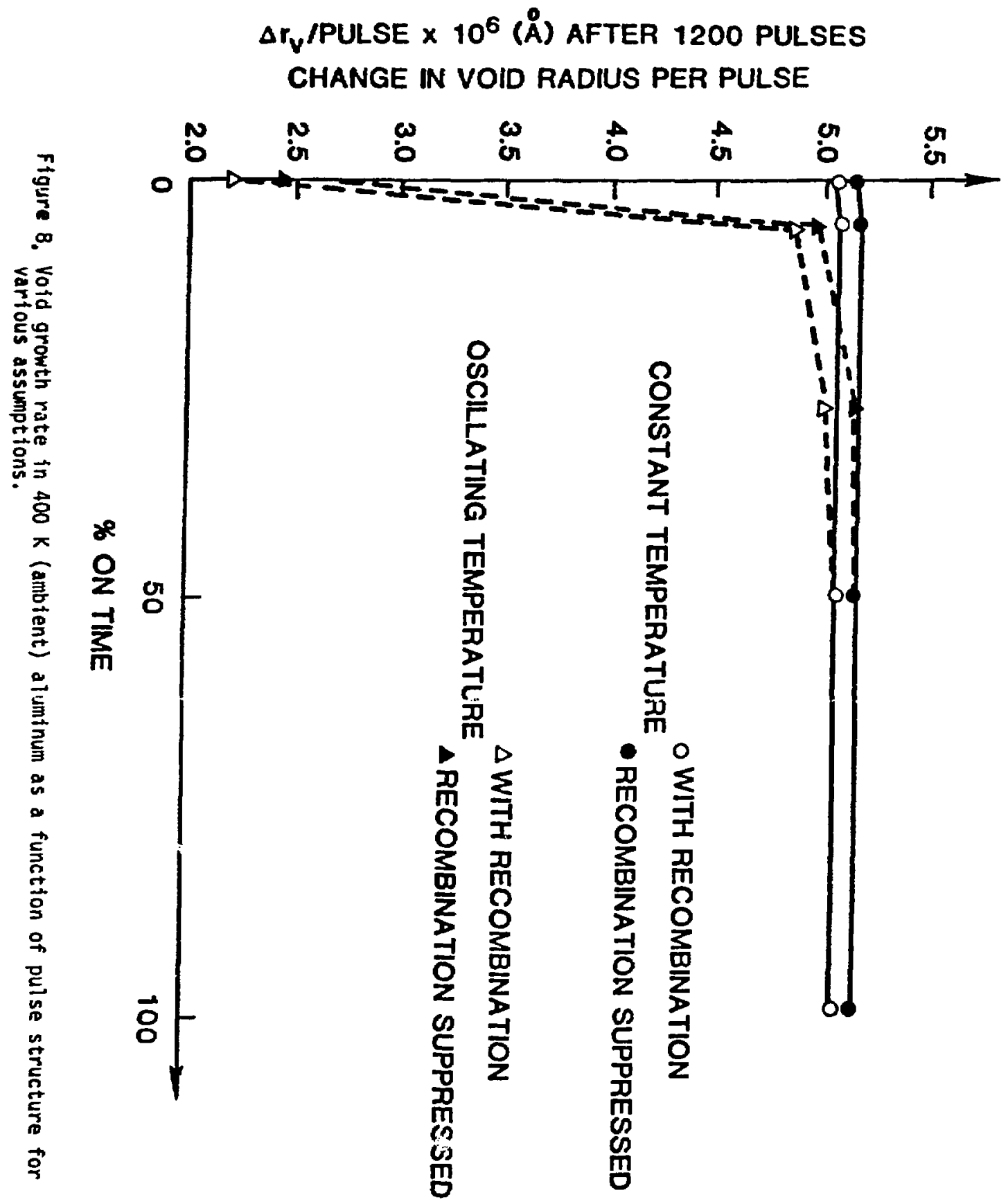




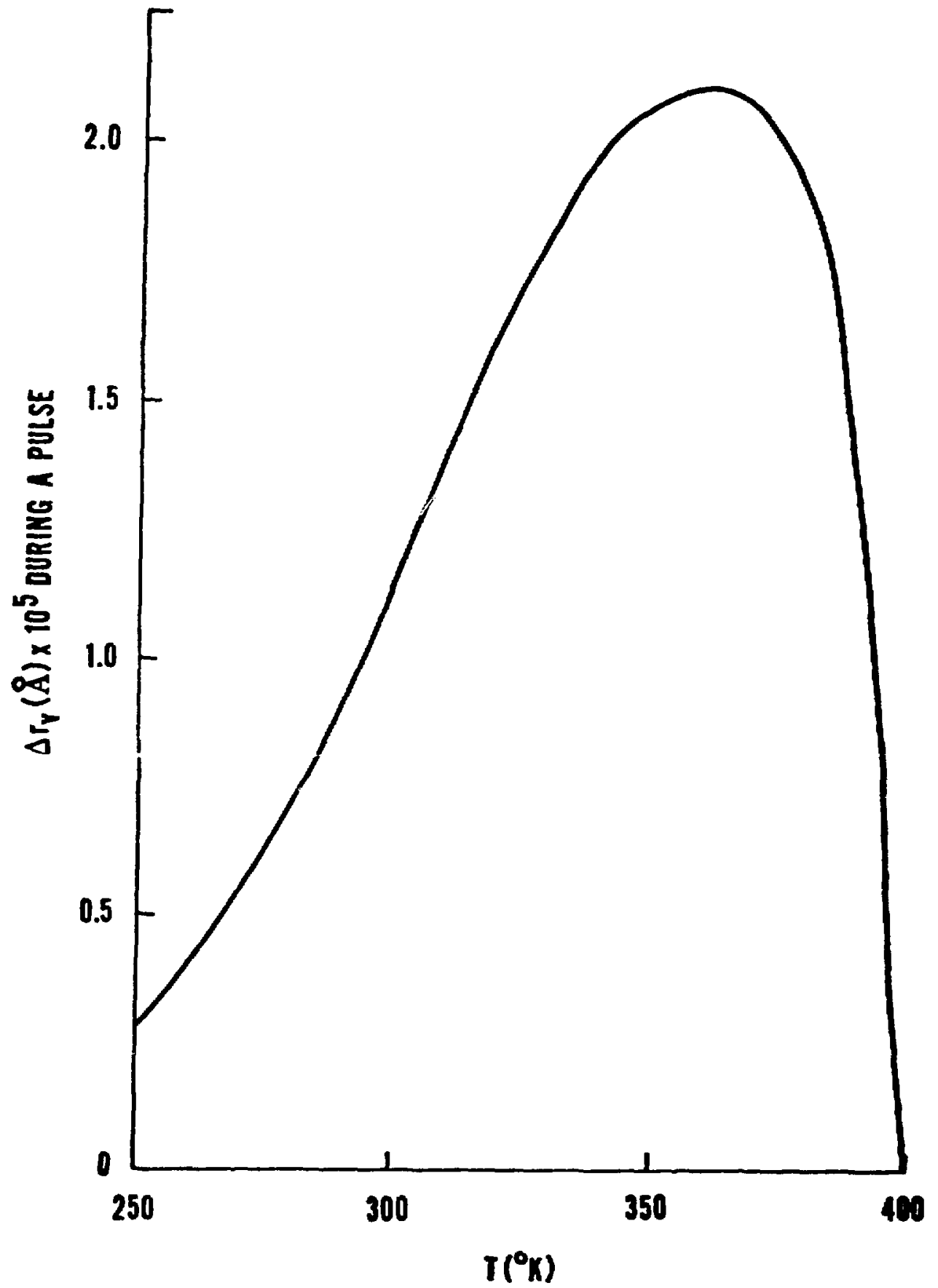

Figure 9. Void growth as a function of ambient temperature during steady irradiation of aluminum. 


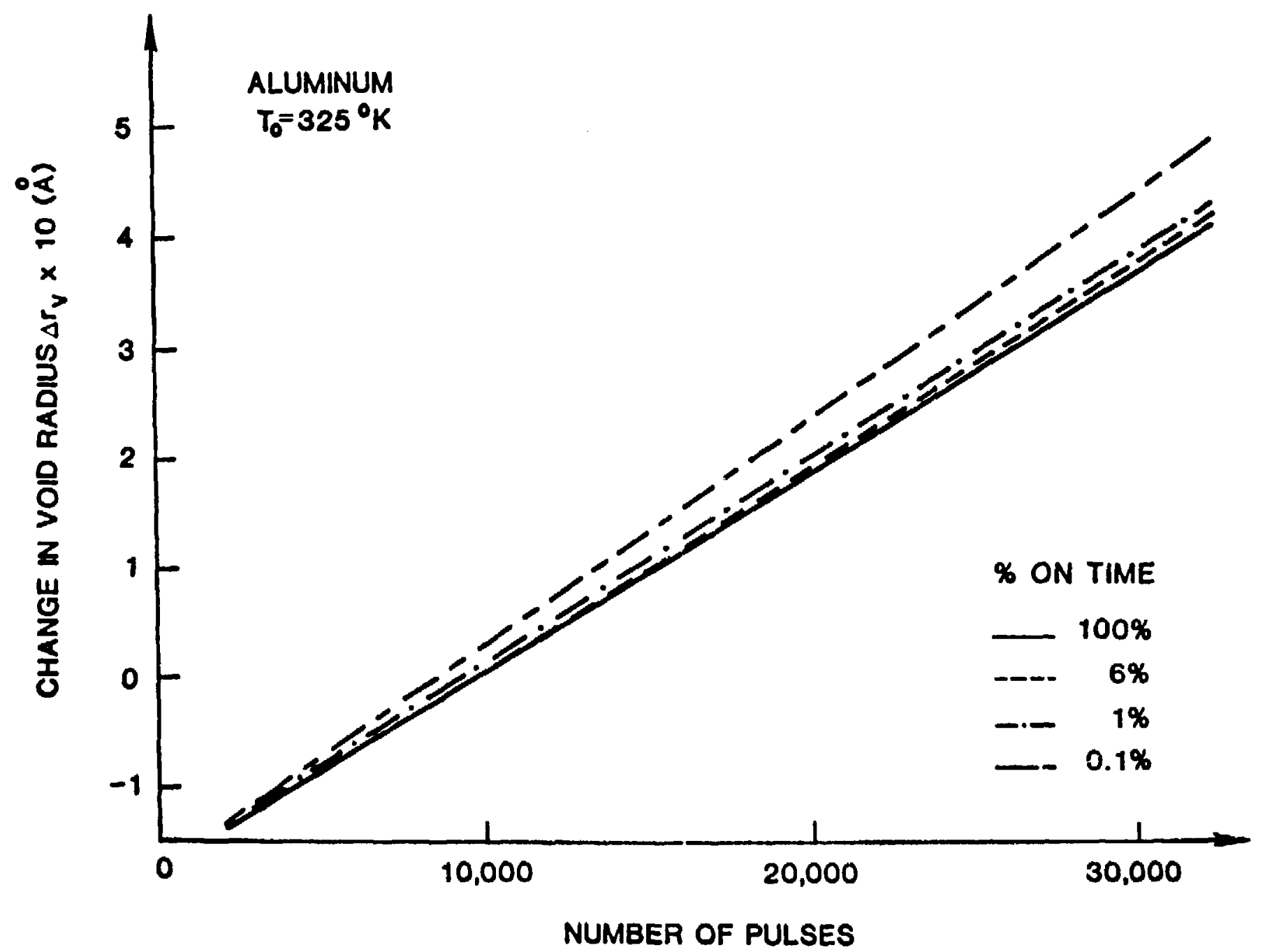


동

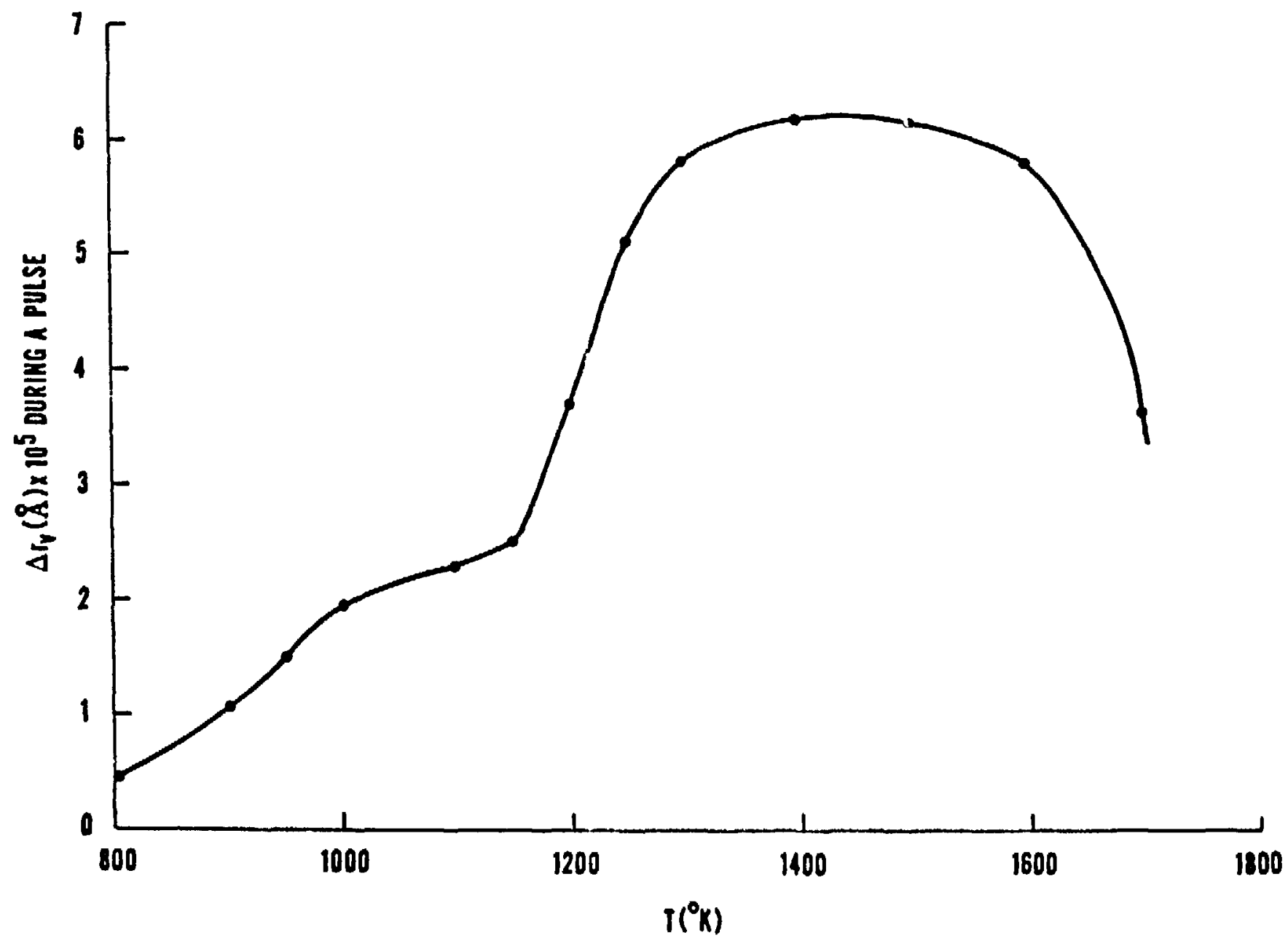

Figure 11. void growth as a function of ambient temperature during steady irradiation of molybdenum. 


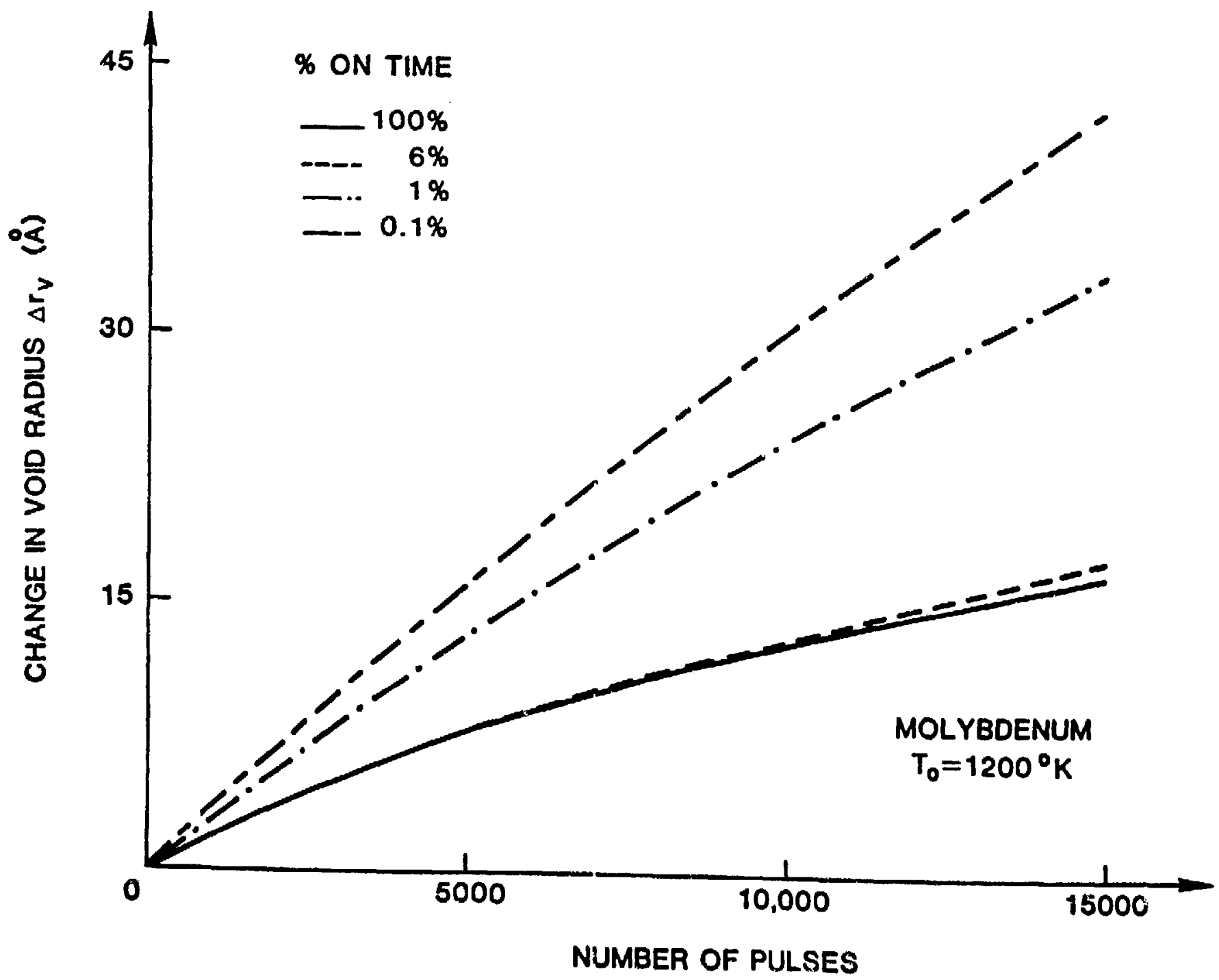

Figure 12. Void growth for various irradiations in molybdenum at an ambient temperature of $1200 \mathrm{~K}$. 


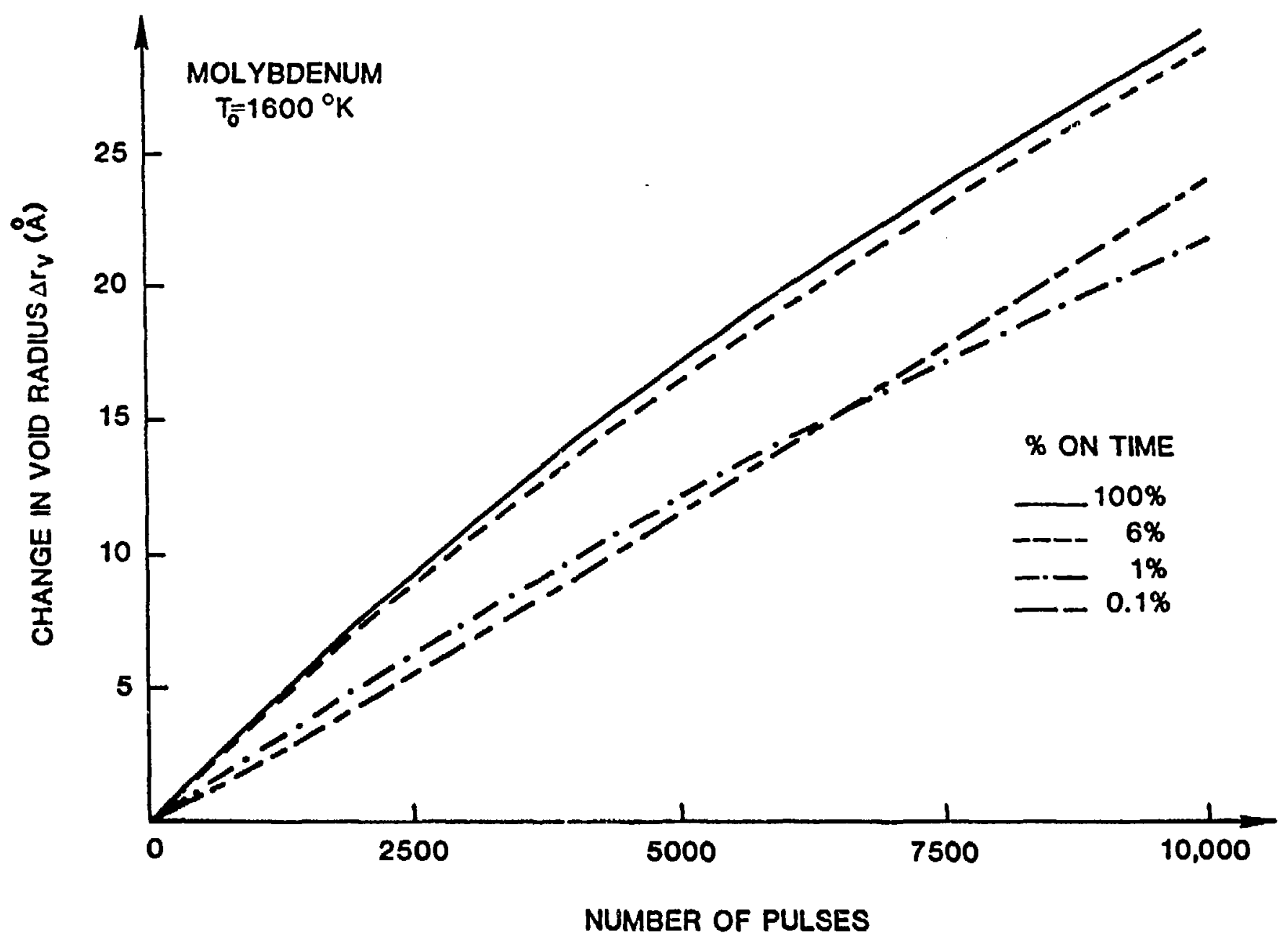

Figure 13. Void growth for various irradiations in molybdenum at an ambient temperature of $1600 \mathrm{~K}$. 


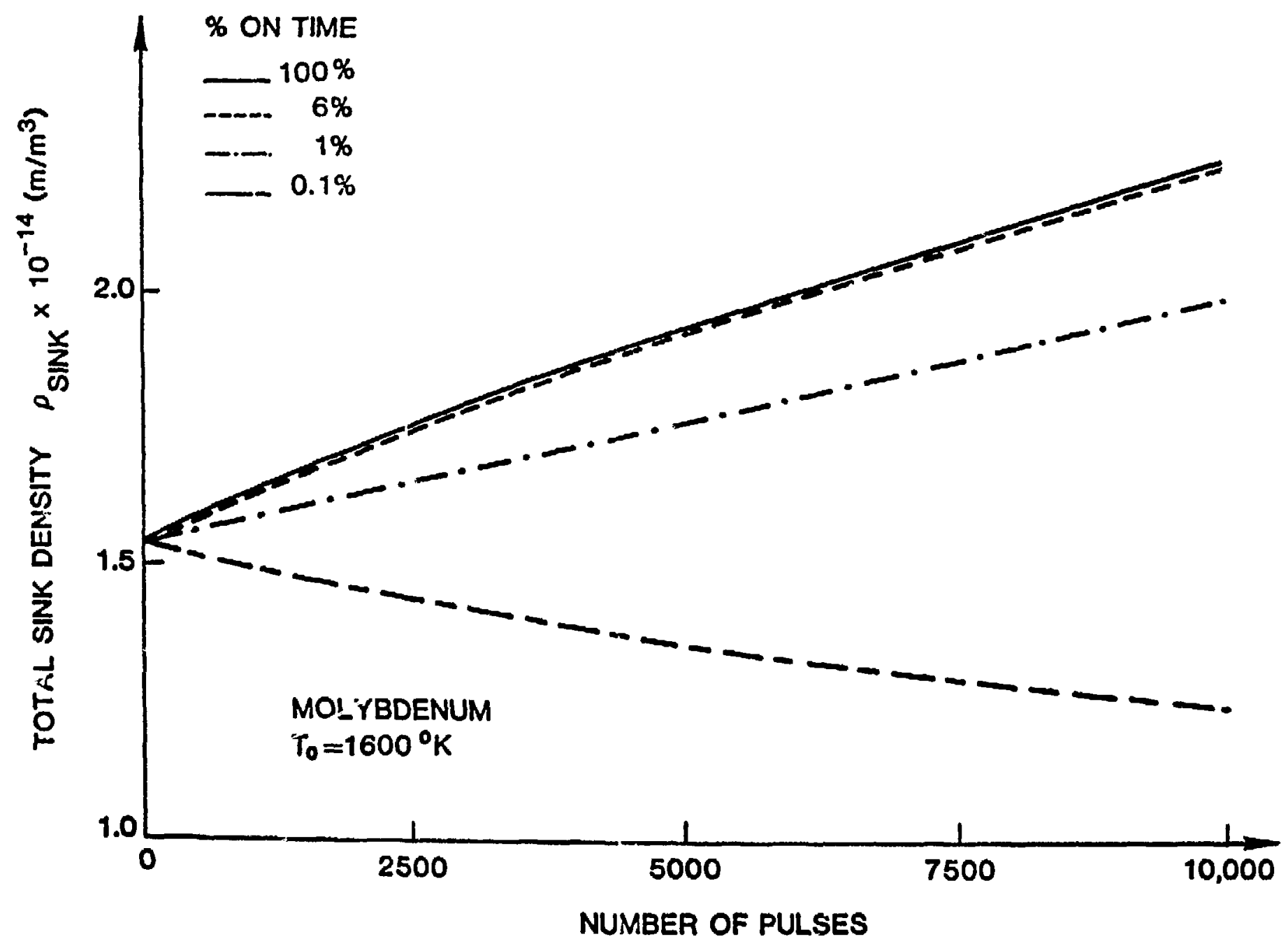

Figure 14. Total sink strengith for various irradiations in molybdenum at an ambient temperature of $1600 \mathrm{~K}$. 

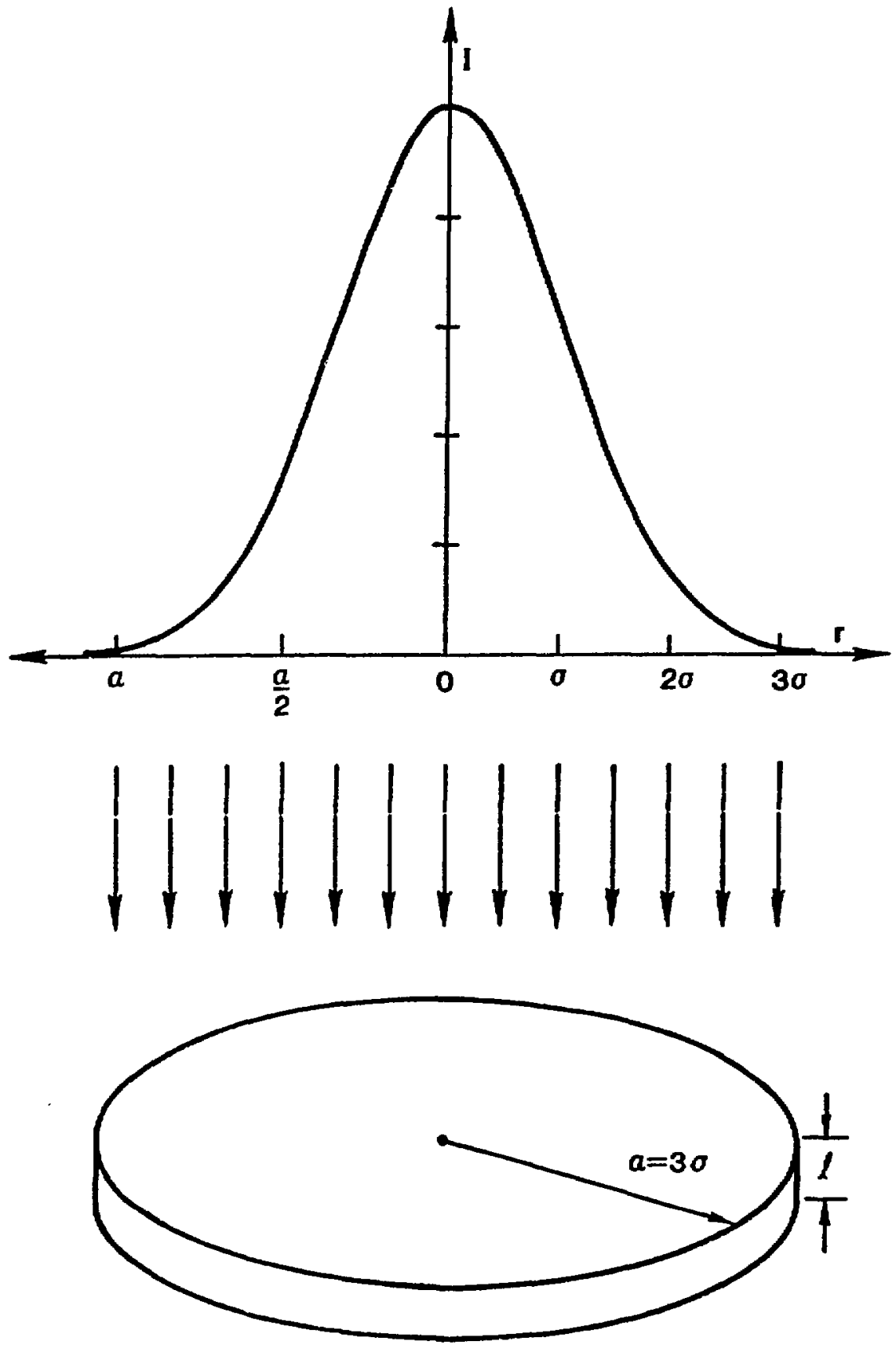

Figure A-1, Gaussian beam structure and target geometry, 


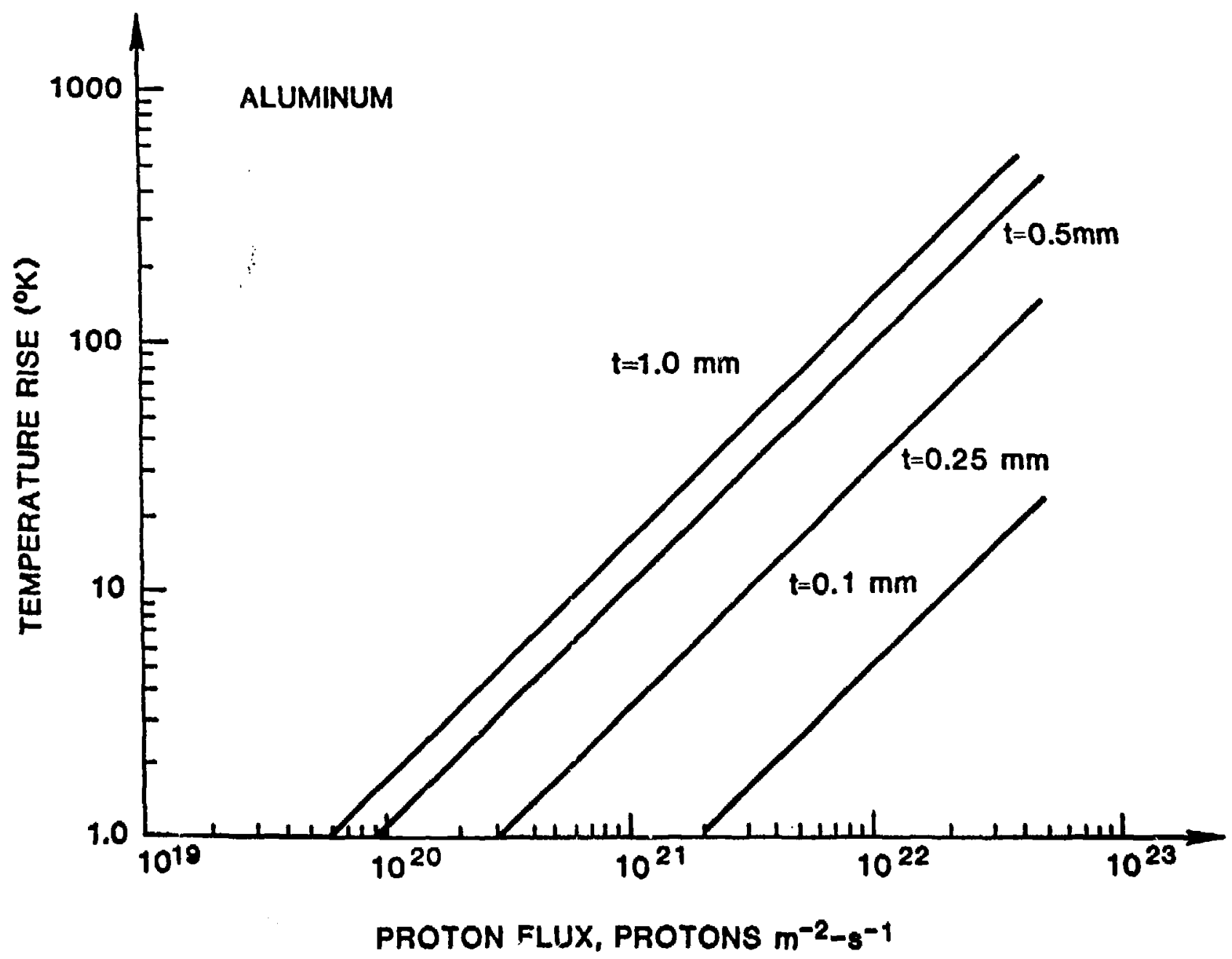




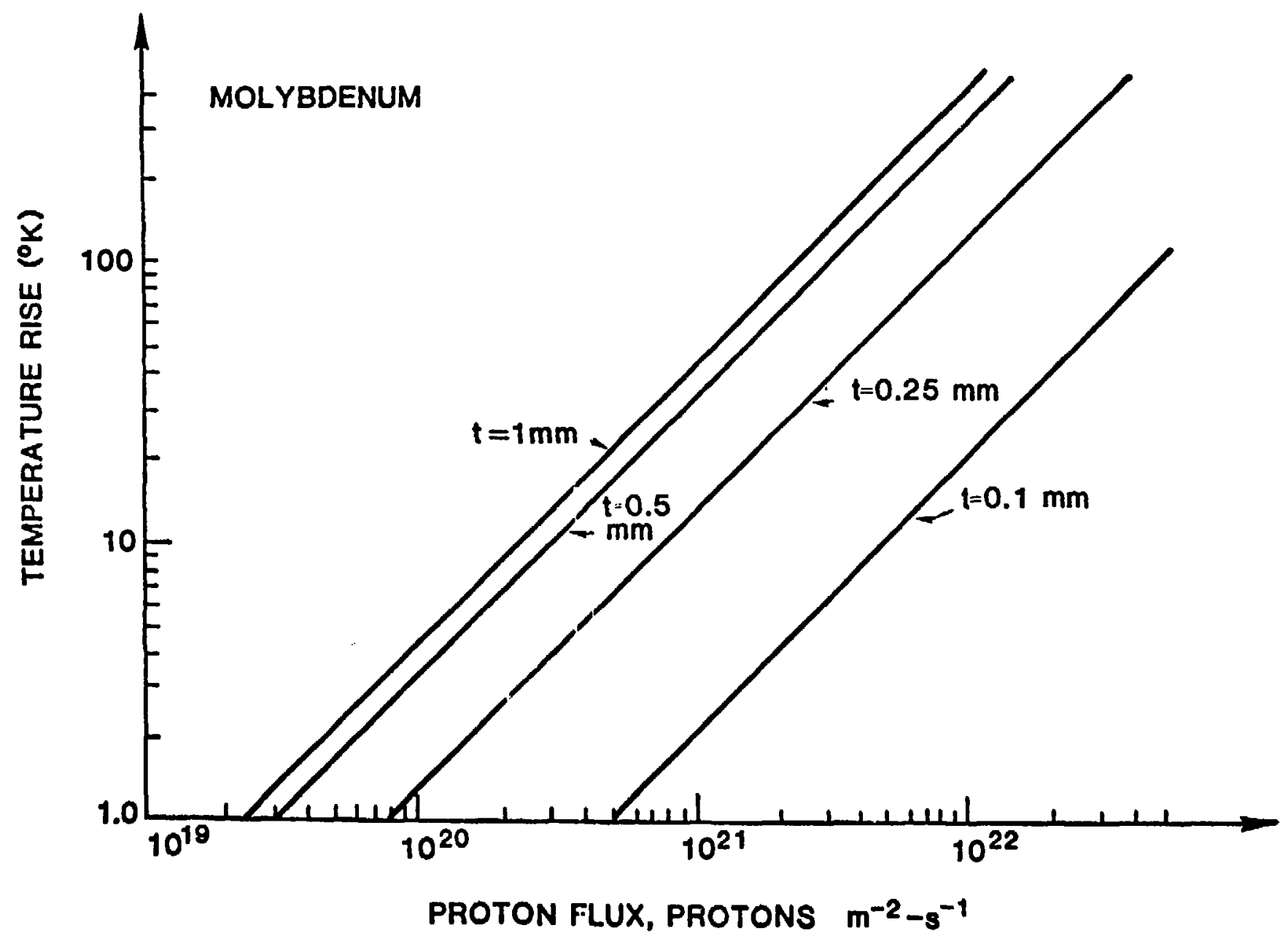

Figure A-3. Temperature rise at the end of a radiation pulse in molybdenum. 


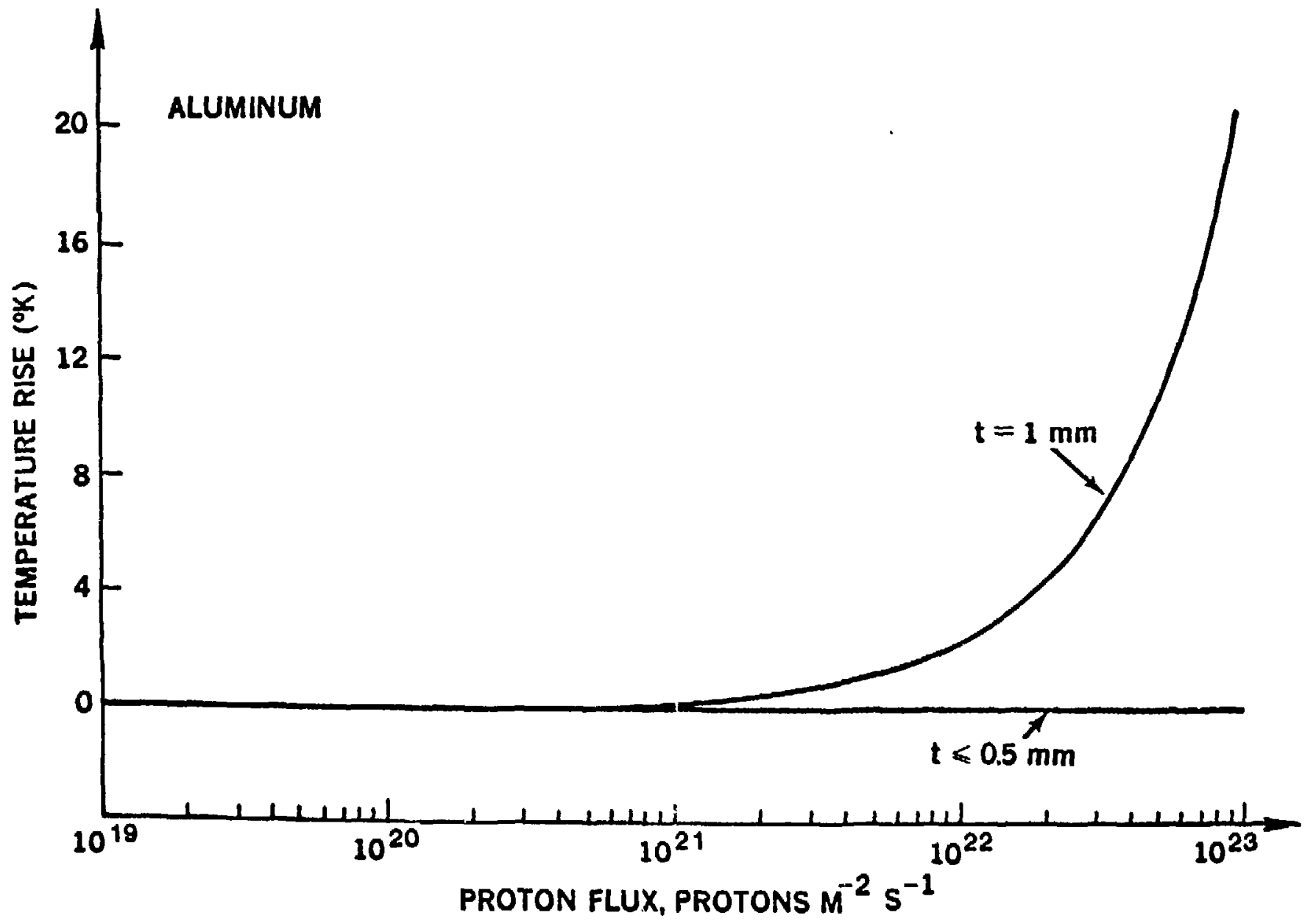


Ч

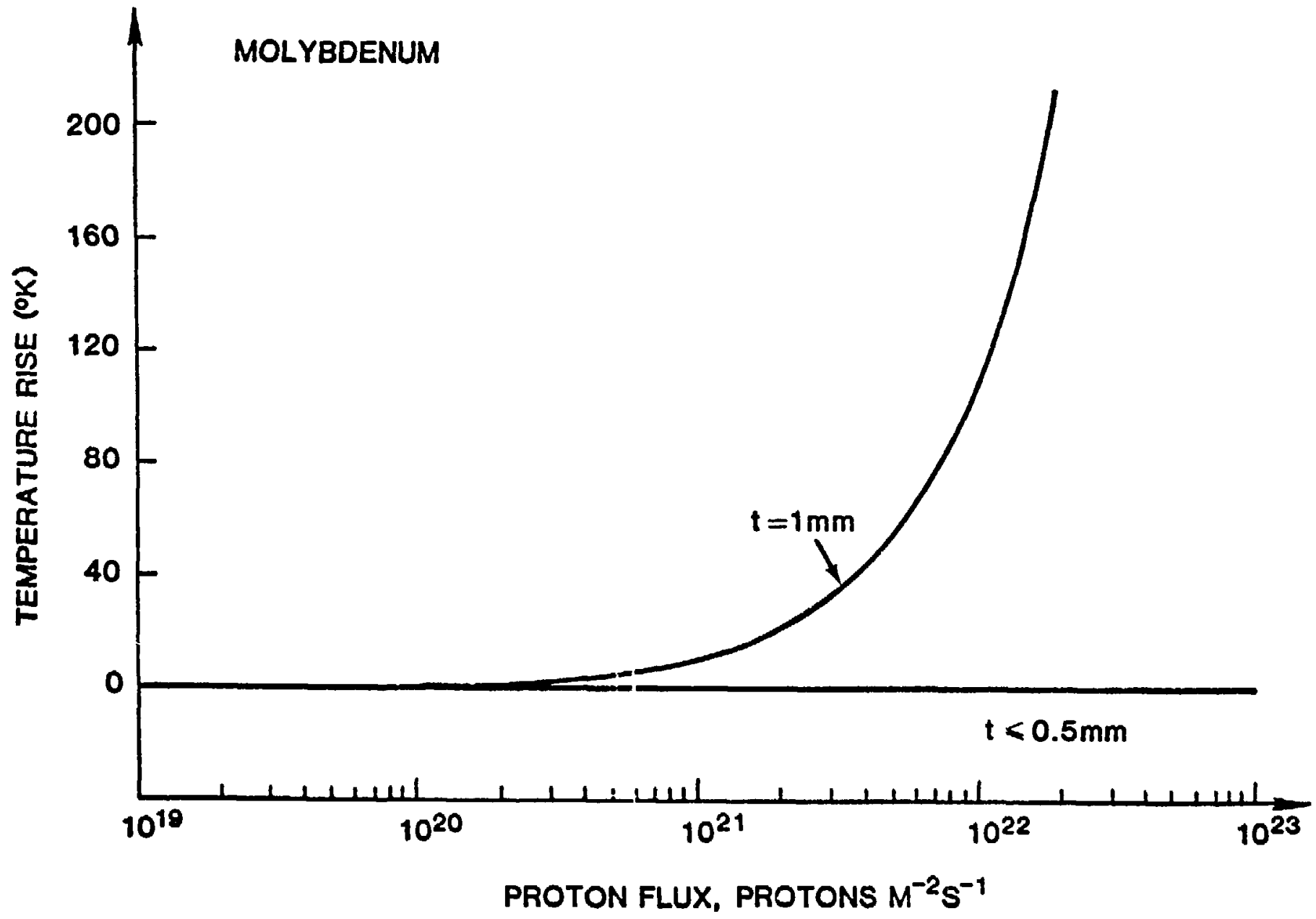

Figure A-5. Temperature rise at the end of an annealing period in molybdenum. 


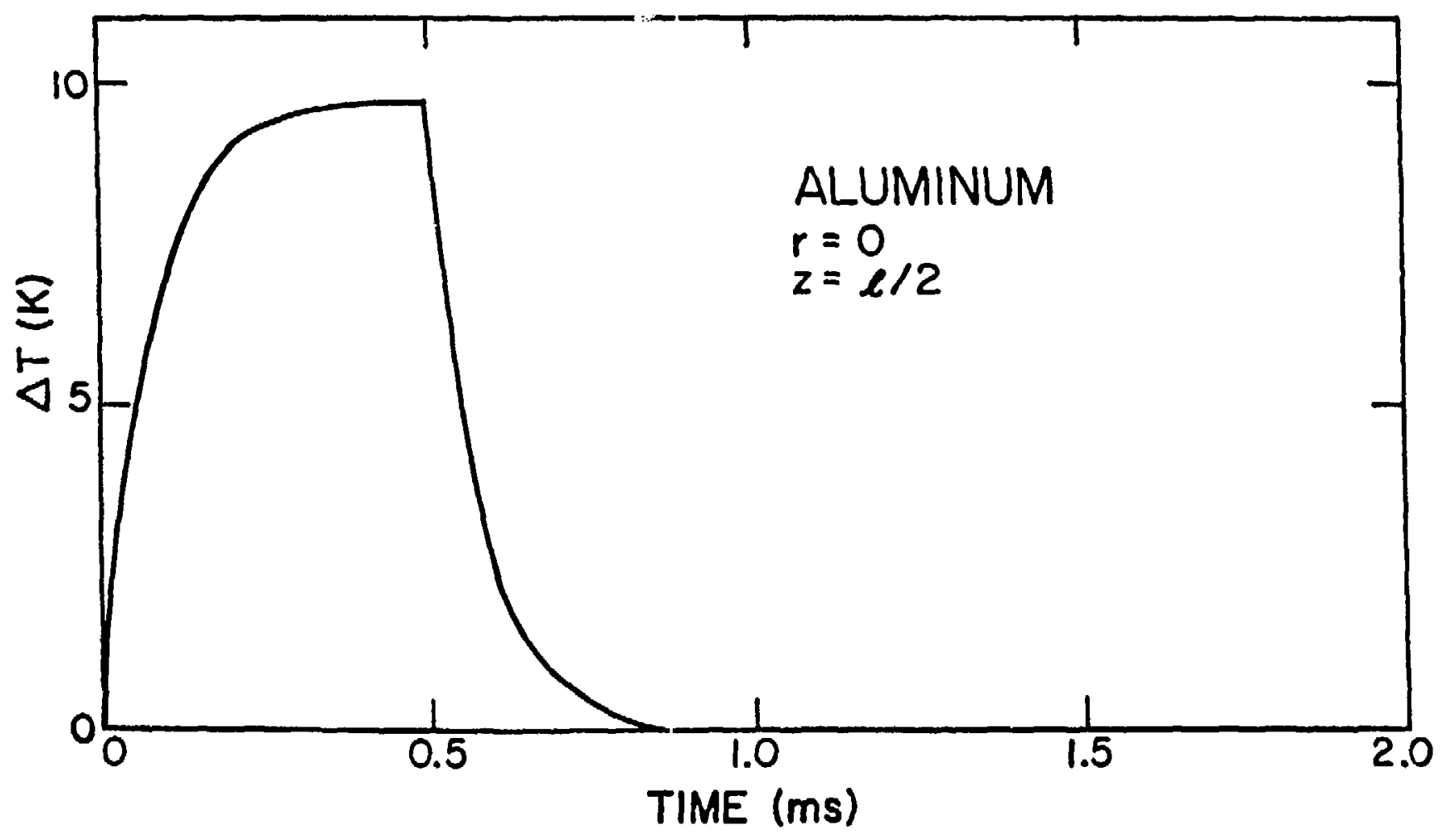

Figure A-6. Temperature response of an aluminum target during the first pulse and annealing period. 


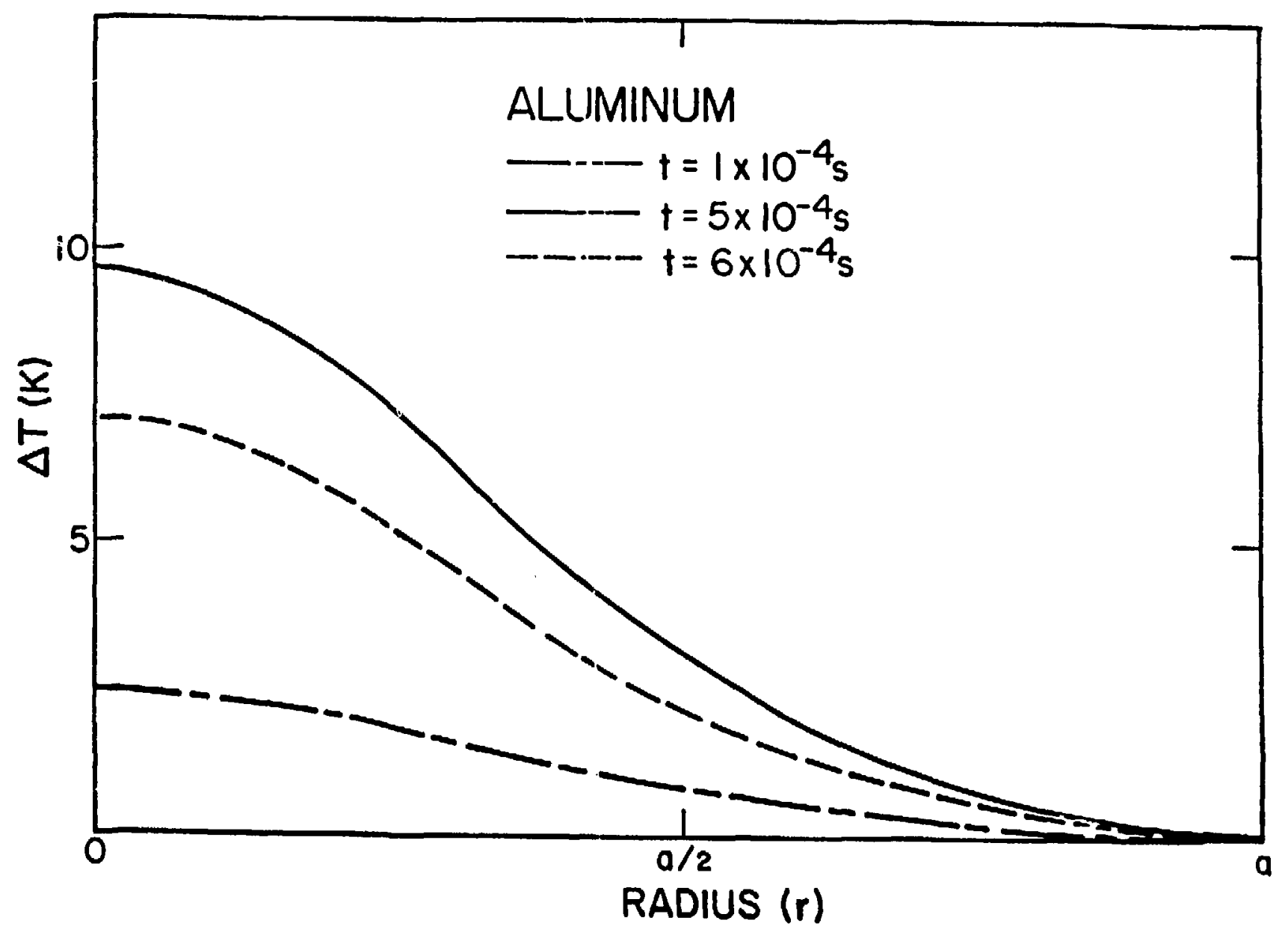

Figure A-7. Radial temperature profiles in aluminum at various times. 


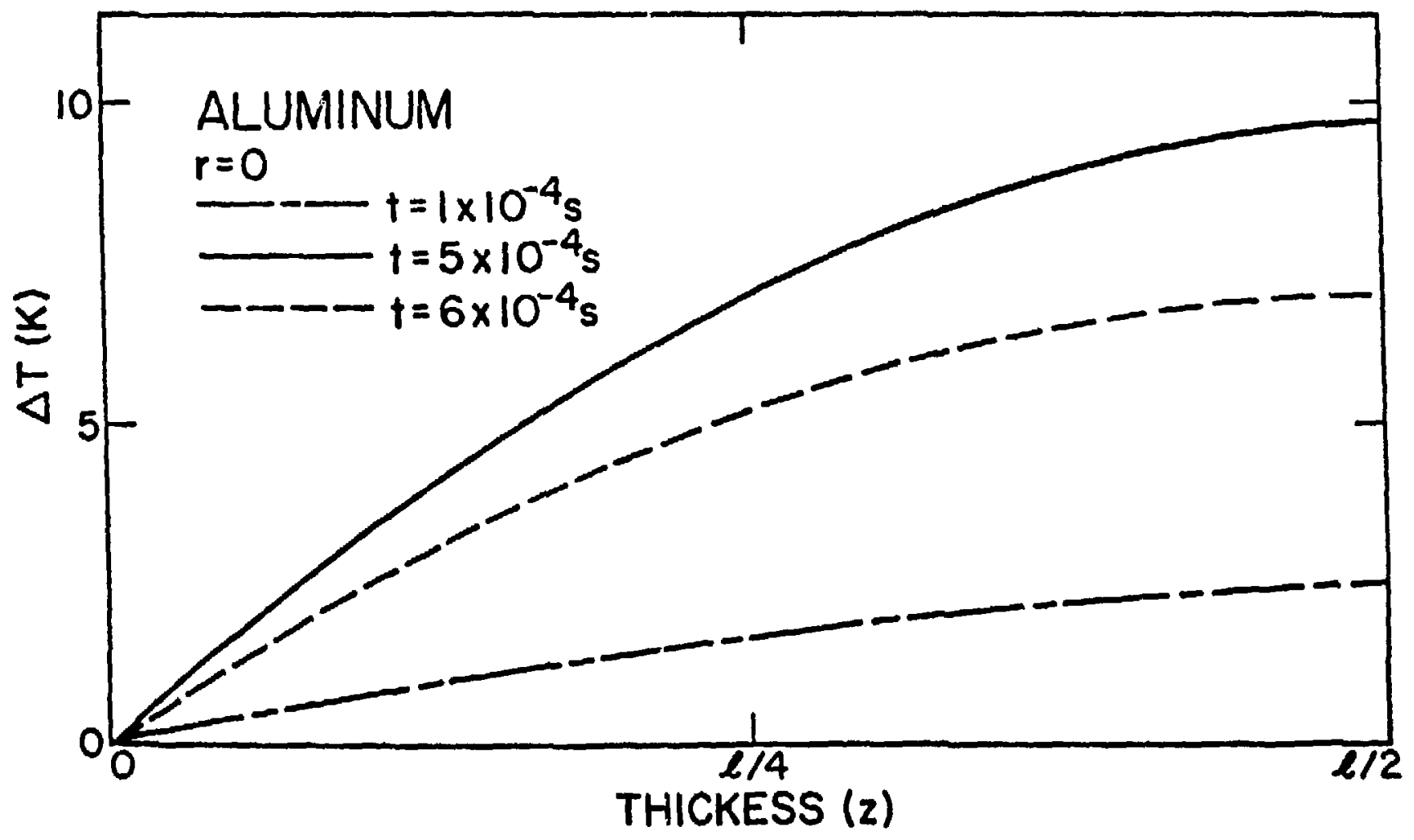

Figure A-8. Axtal temperature profiles in aluminum at various times. 
옹

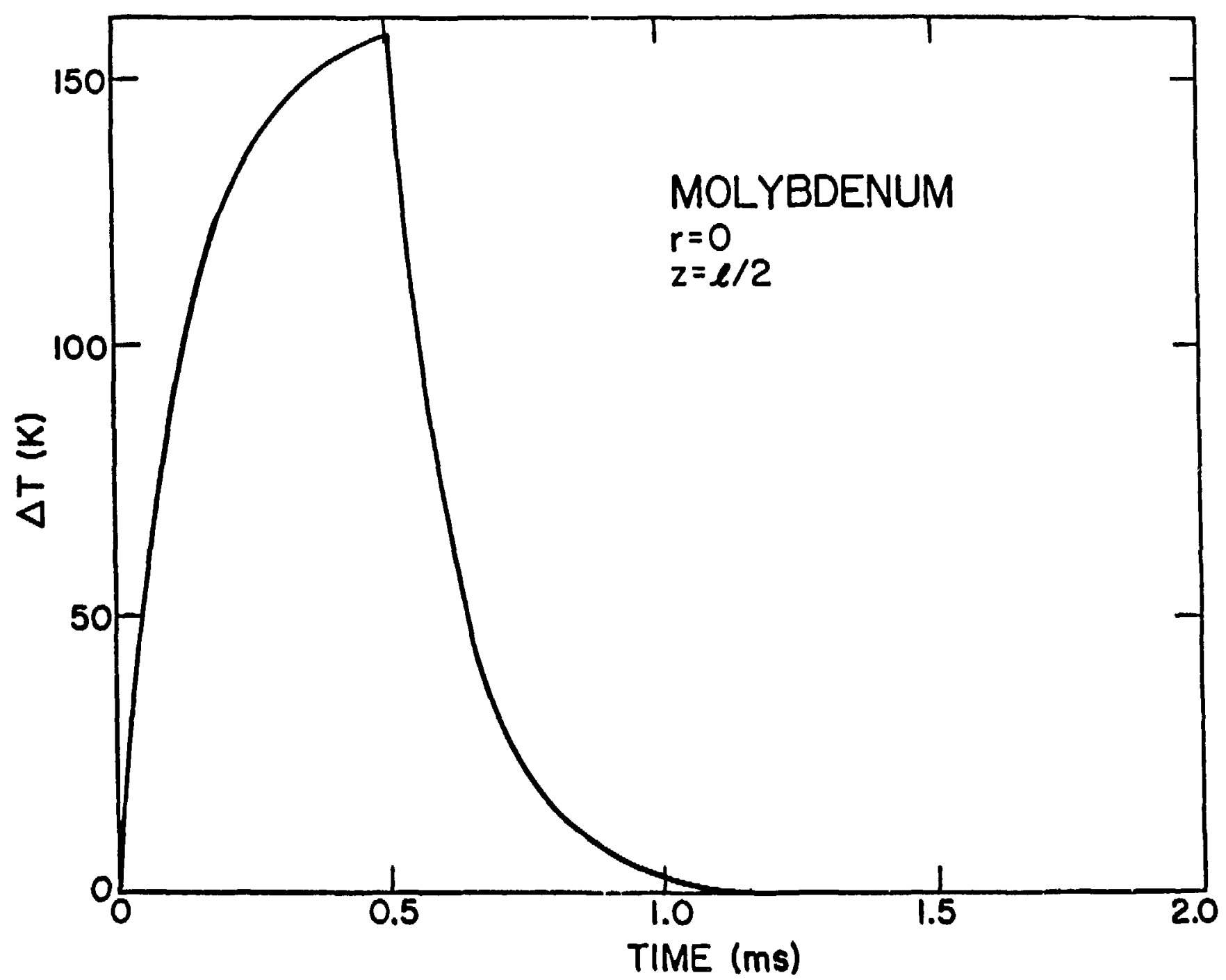

Figure A-9.Temperature response of molybdenum tirget during the first pulse and annealing period. 


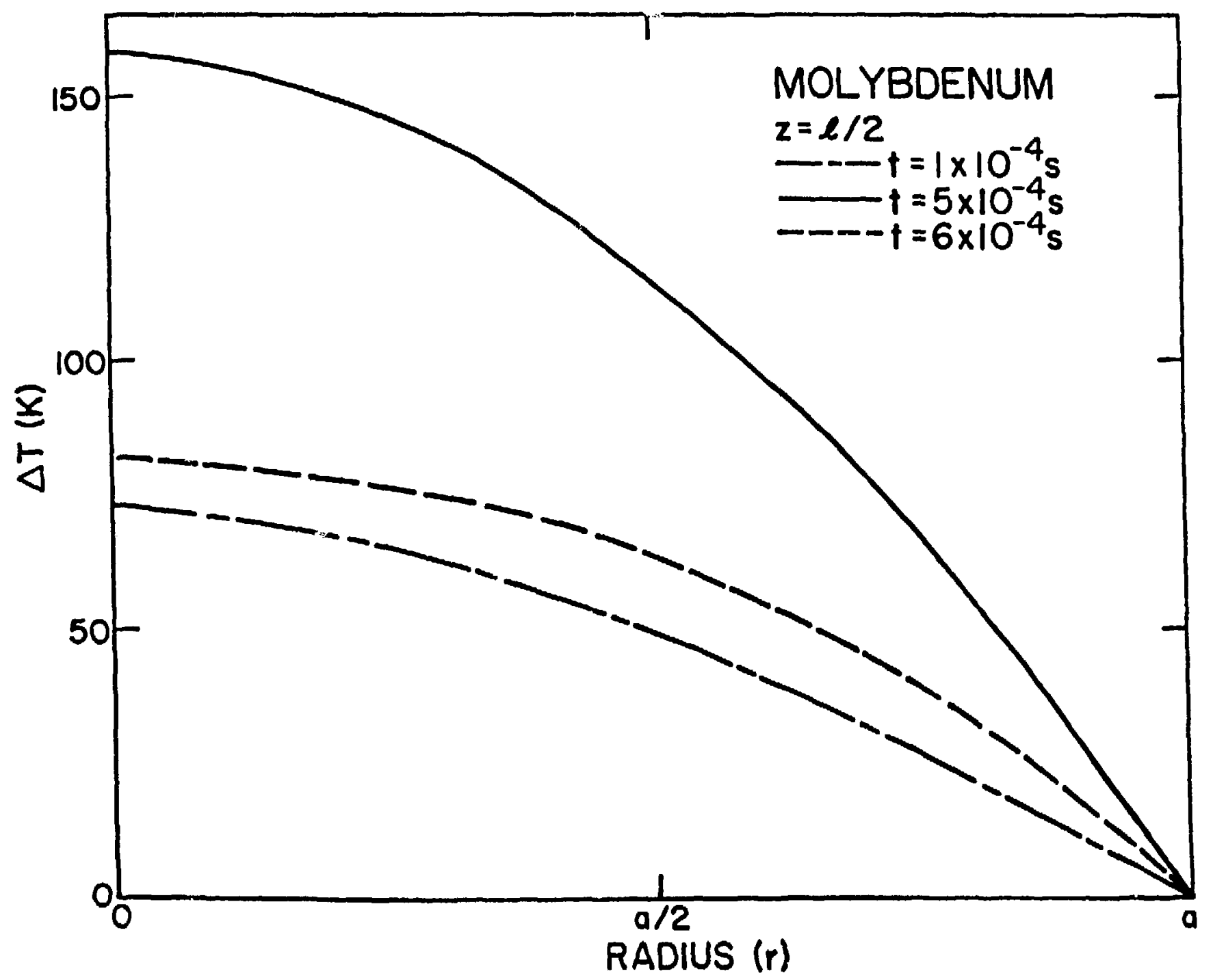




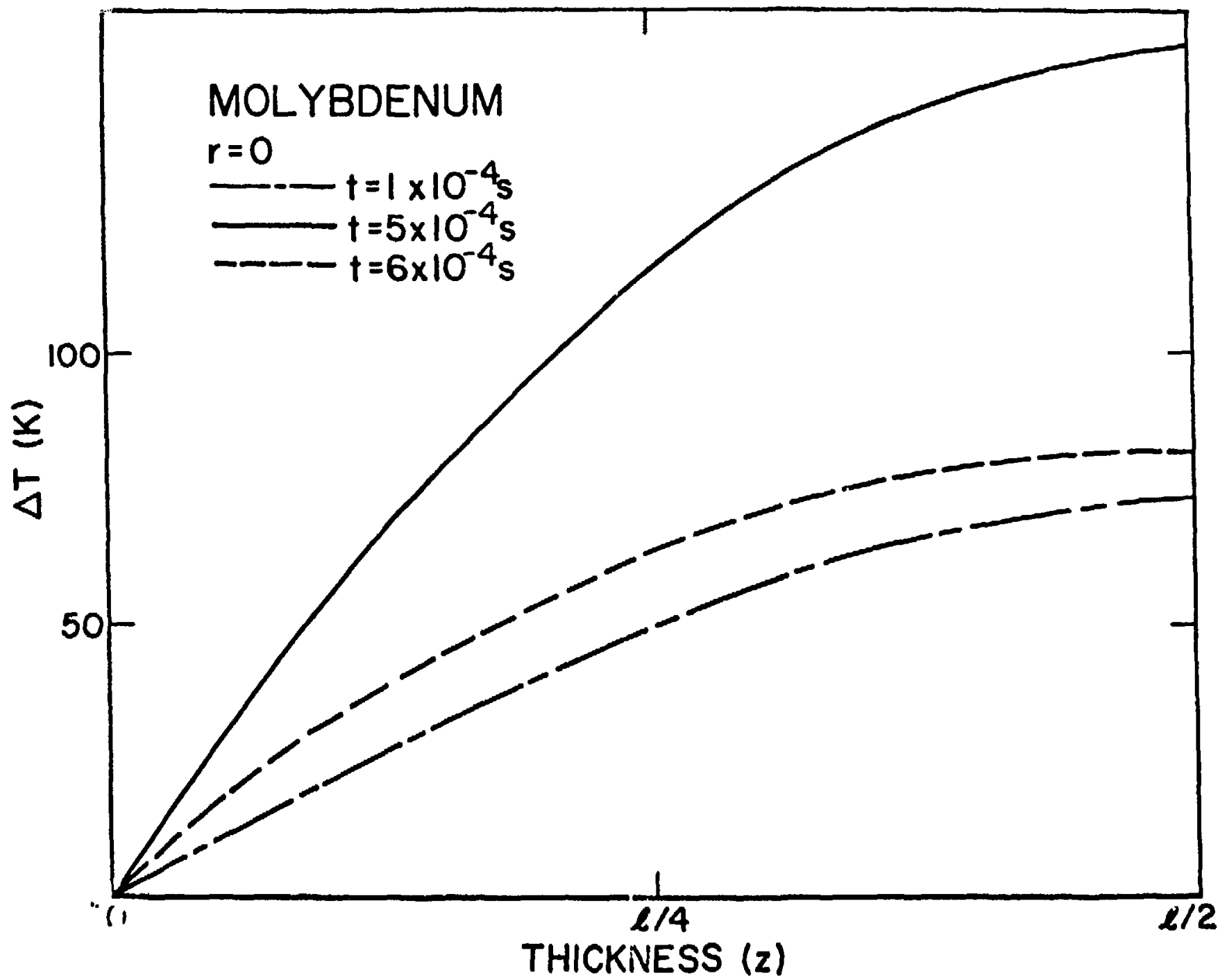

Figure A-11. Axial temperature proflles in molytidenum at various times. 


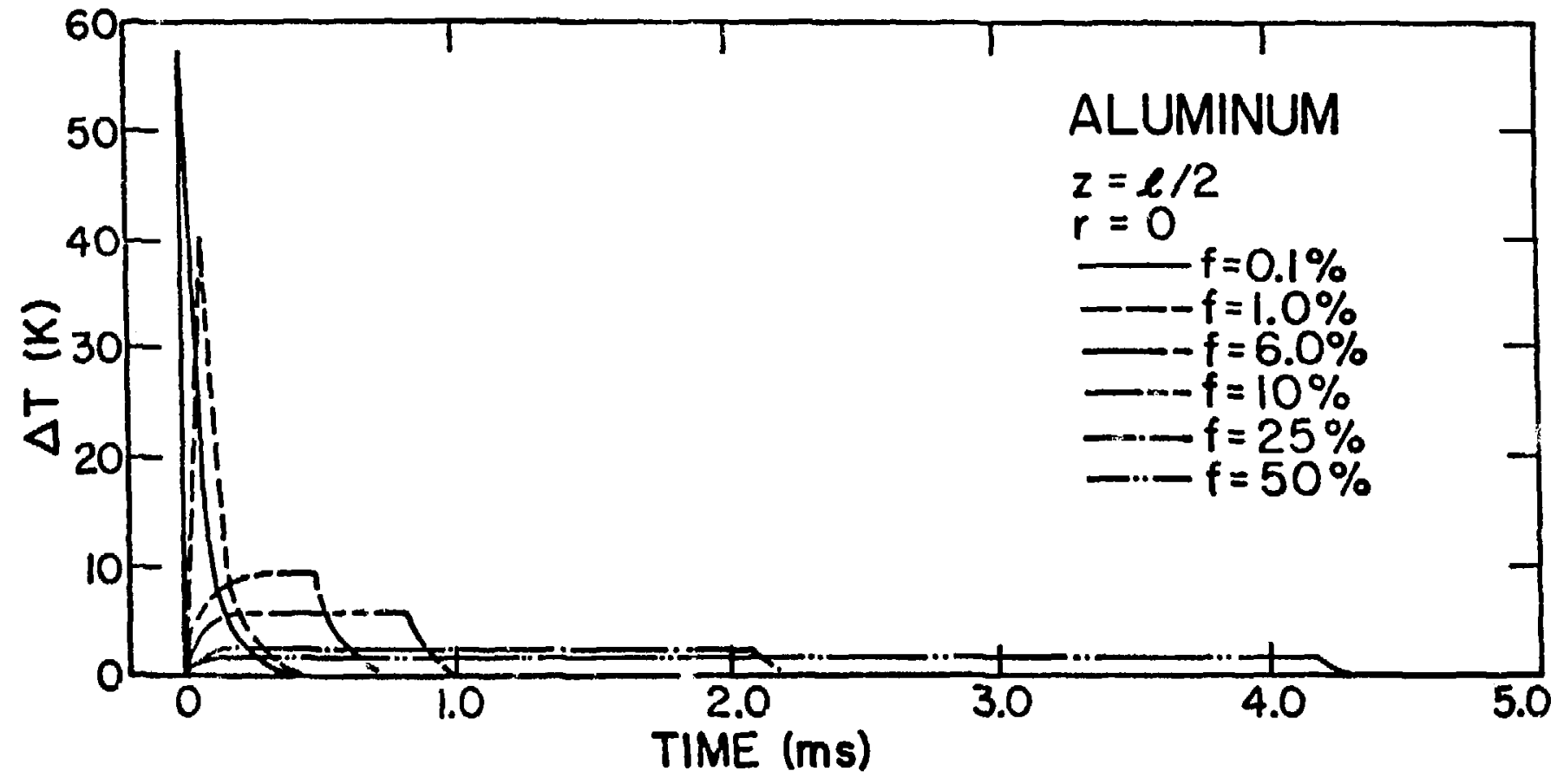




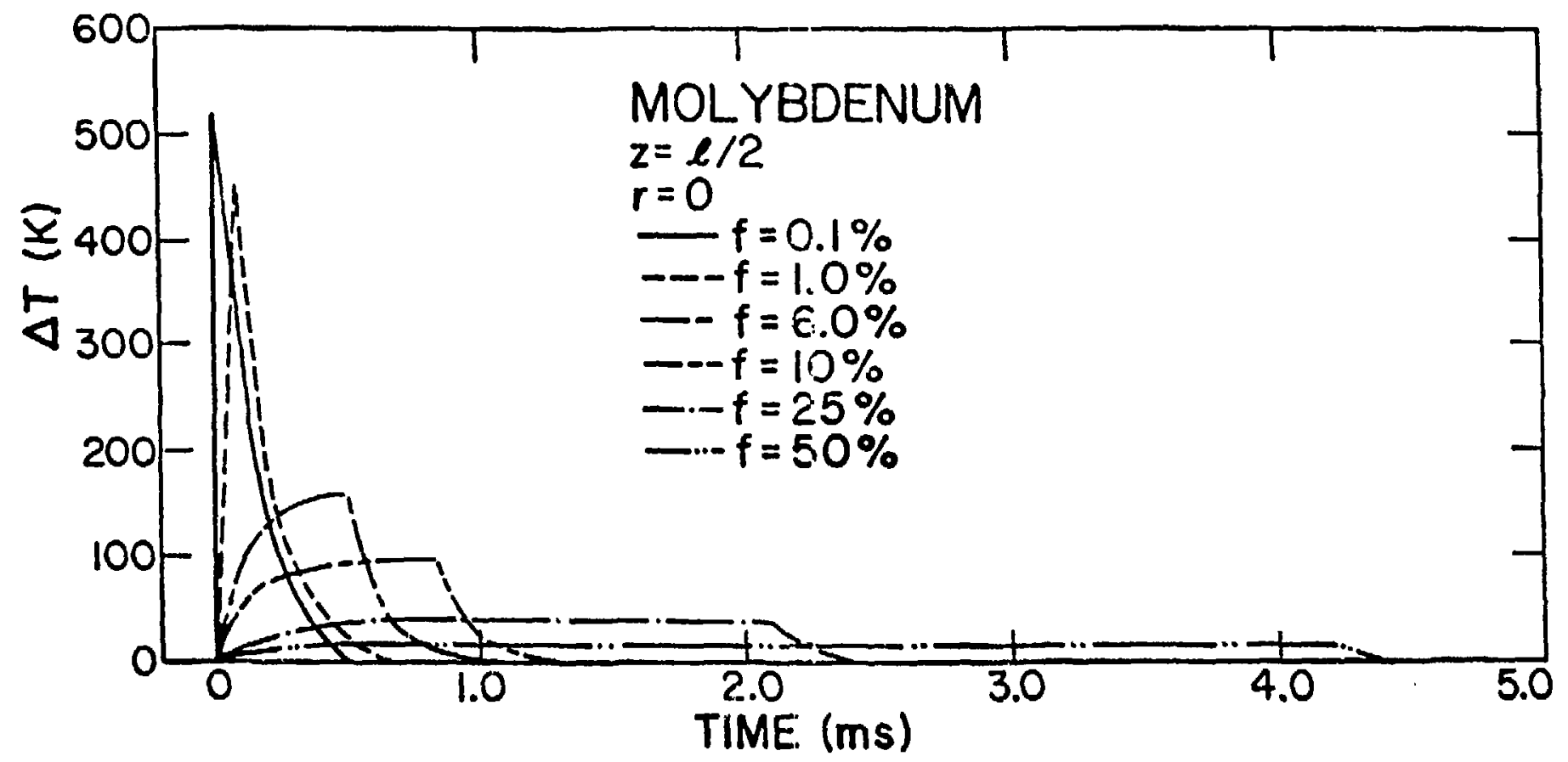

Figure A-13. Temperature response of molybdenum to various pulse structures. 


\section{REFERENCES}

1. A. D. Brailford and R. Bullough, J. Nucl. Mat. 44, (1972) p. 121.

2. A. D. Brailford and R. Bullough, Proc. Physical Metallurgy of Reactor Fuel Elements, Gloucestershire 1973 (eds. J. E. Harris and E. C. sykes), 148; 1975 Metals Society.

3. R. Bullough, B. L. Eyre and R. Krishan, Proc. R. Soc. Lond. A. 346, (1975) p. 31 .

4. N. Ghoniem and G. Kulcinski, Fully Dynamic Rate Theory (FDRT) of Radiation Induced Swelling of Metals, Univ. of Wisc. UWFDM-180 (1976).

5. N. Ghoniem and G. Kulcinski, TRANSWELL (Version 1): A Computer Code for Metal Swelling and Creep under Transient, Pulsed or Steady State Conditions, Univ. of Wisc. UWFDM-181 (1976).

6. N. Ghoniem and G. Kulcinski, Calibration of the Fully Dynamic Rate Theory Using the Computer Code TRANSWELL, Univ. of Wisc. UWFDM-200 (1977).

7. N. Ghoniem and G. Kulcinski, The Importance of Materials and Irradiation Parameters on Void Growth in Metals - Part I - The Effect of Displacement Rate and Cascade Efficiency, Univ. of Wisc. IJWDM-203 (1977).

8. G. J. Dienes, Rad. Effects 36 (1978) p. 101.

9. C. A. Coulter, D. M. Parkin and W. V. Green, J. Nucl. Mat. (1977) P. 140.

10. C. W. Gear, Numerical Initial Value Problems in Ordinary Differential Equations, Prentice-Ha11, Englewood Cliffs, NJ (1971).

11. A. C. Hindmarsh, GEAR: Ordinary Differential Equaition Solver, Lawrence Livermore Laboratory UCID-3000001, Rev. 3 (1974).

12. C. D. Sutherland and D. Kahaner, SDRIVE: Ordinary Differential Equation Equation Solver, Los Alamos Scientific Laboratory PIM-2 Program Library Writeup D207 (1977).

13. H. S. Carslaw and J. C. Jaeger, Conduction of Heat in Solids, CTaredon Press, Oxford (1959). 